\title{
Asteroid flyby opportunities using semi-autonomous CubeSats: mission design and science opportunities
}

\author{
P. Machuca ${ }^{\mathrm{a}, \boldsymbol{}}$, J. P. Sánchez ${ }^{\mathrm{a}}$, S. Greenland ${ }^{\mathrm{b}}$
}

a Space Research Group, Cranfield University, Cranfield, United Kingdom

${ }^{\mathrm{b}}$ Craft Prospect Ltd, Glasgow, United Kingdom

\begin{abstract}
CubeSat technology has recently attracted great interest from the scientific community, industry and space agencies, and represents today an exciting movement towards a more affordable and accessible space industry. In view of potential applications of CubeSat technology to small-body planetary exploration, this paper studies the feasibility of using autonomous CubeSats to flyby near-Earth asteroids. This work provides an overview of the current state of CubeSat technology and proposes a 3U CubeSat mission using primarily off-the-shelf components. The proposed mission considers a CubeSat is deployed by a larger spacecraft in a periodic orbit around the first (L1) or the second (L2) Sun-Earth Lagrange points (common destinations to observe the Sun and outer space), from where fuel-optimal impulsive trajectories are designed to flyby asteroids between 2019 and 2025. Navigation support and ground operations costs still represent a major challenge for interplanetary CubeSats. As such, Monte Carlo simulations are performed to determine the flyby accuracies that can be accomplished by a $3 \mathrm{U}$ CubeSat flying autonomously (i.e., using observations of the Sun during cruise and observations of the asteroid before the flyby to estimate its own trajectory, instead of using ground stations for navigation support). Asteroid flyby opportunities for an autonomous 3U CubeSat are identified between years 2019 and 2025. Flyby altitudes below $500 \mathrm{~km}$ are found possible with currently-available CubeSat components. Possible science payloads are also overviewed, and the potential scientific return of such a low-cost mission is discussed.
\end{abstract}

\section{Keywords:}

Interplanetary CubeSats

Near-Earth asteroids

Trajectory design

Autonomous navigation

\section{Introduction}

Miniature spacecraft the size of a shoebox (known as CubeSats - see Fig. 1) have gathered significant interest amongst the scientific community, industry, and space agencies over the past decade. In an effort to transform space exploration into a more affordable and accessible industry, CubeSats offer a low-cost alternative to traditional space missions and have promoted the standardization and miniaturization of spacecraft components.

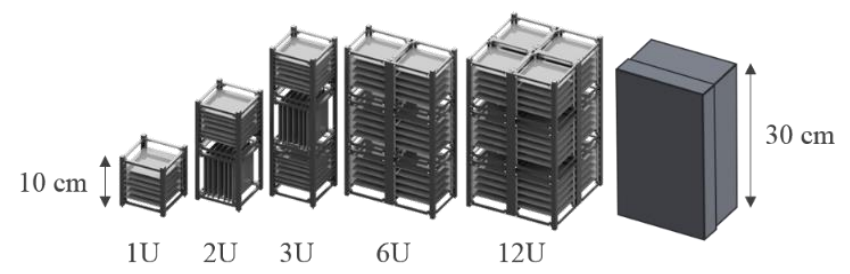

Fig. 1. CubeSat $1 \mathrm{U}-12 \mathrm{U}$ structures (modified from Radius Space, 2016).

CubeSats come in different sizes (all of which are composed of $10 \mathrm{~cm} \times 10 \mathrm{~cm} \times 10 \mathrm{~cm}$ cubical units- denoted as 1U), and although their capabilities are limited by their small mass and volume, they are currently employed in low-Earth orbit (LEO) for communication purposes and for Earth observation (Aslan et al., 2013; Jove-Casulleras et al., 2012; Praks et al., 2015).

The CubeSat industry is rapidly evolving, and easilyimplemented, ready-to-use components are now produced by a variety of manufacturers. These commercial off-the-shelf components allow for a cost reduction in the development and implementation of CubeSats, ultimately enabling space missions with budgets 5-10\% those of traditional missions (Heidt et al., 2000; Selva and Krejci, 2013).

As such, a current trend in the space community is conceptualizing the potential applications and novel mission architectures now enabled by these miniature spacecraft (Poghosyan and Golkar, 2017). Proposed applications for CubeSats include biological experiments and astronomical observations from LEO, the observation and study of the Moon, supporting larger spacecraft

\footnotetext{
* Corresponding author.

E-mail address: p.machuca@cranfield.ac.uk (P. Machuca).
} 
missions, the exploration of comets, asteroids, planets and other planetary bodies, etc.

Proposed CubeSat missions beyond LEO generally consider deploying CubeSats from a launch vehicle or a larger spacecraft that is already in space (Hunter, 2015). In view of their limited propulsion capabilities, these socalled "piggyback" opportunities represent the most viable option for CubeSats to reach farther destinations. CubeSats have been successfully deployed from the International Space Station (ISS) and NASA's Atlas V launcher (Pournelle, 2014; Schoolcraft et al., 2016), and will soon be launched by NASA's new Space Launch System (SLS) (Singer et al., 2017). Although numerous and varied missions have been proposed, only one mission is planned to flyby an asteroid using CubeSats: the Near-Earth Asteroid (NEA) Scout mission (a 6U, <12kg CubeSat mission), which will be launched along NASA's SLS in 2019 (Mcnutt et al., 2014).

In response to the current interest in understanding the potential of CubeSats (e.g., how far they can go, what kind of science they can achieve, how small missions can be, etc.), this work studies the feasibility of flying by a near-Earth asteroid using a $3 \mathrm{U}$ CubeSat (i.e., $<4 \mathrm{~kg}$ ), evaluates the flyby altitudes that can achieved, and discusses the potential scientific impact of such a mission.

In addition to investigating whether asteroid exploration is possible using smaller CubeSats, this work also analyzes the possibility of performing autonomous navigation and guidance instead of using ground segment operations to estimate the position and velocity of the spacecraft, and to correct its trajectory-which generally requires extensive workforce and the use of large and costly ground stations such as the Deep Space Network (DSN). Although the development cost of CubeSat missions is highly reduced in comparison to larger missions, ground operations costs do not scale down proportionally to spacecraft size and still represent a very significant - sometimes inviable - fraction of mission budgets (Cheung et al., 2015).

If the study of asteroids is demonstrated to be possible using autonomous $3 \mathrm{U}$ CubeSats, these could provide a low-cost solution to determine initial stages of solar system formation through asteroid exploration (or the primordial sources of organic matter if used for cometary exploration) (National Research Council, 2013), they could be used to identify potential targets for future missions, to complement the scientific objectives of larger missions, or to support the emerging asteroid mining industry (Calla et al., 2017), etc. Noteworthy small-satellite missions proposed by commercial asteroid mining companies include, for instance, Deep Space Industries' $50-\mathrm{kg}$ microsatellite mission to land on an asteroid (Bonin et al., 2016), and Planetary Resources' alreadyin-orbit $6 \mathrm{U}$ CubeSat mission to demonstrate asteroid prospecting technologies (Planetary Resources, 2018).

The following sections describe the proposed asteroid exploration mission concept (Section 2), provide an overview of the current state of CubeSat technology and available off-the-shelf components, and propose a $3 \mathrm{U}$ CubeSat design (Section 3), explain the trajectory design and target selection process (Section 4), study the feasibility of performing autonomous navigation and determine attainable asteroid flyby altitudes (Section 5), and assess the potential scientific return of the mission (Section 6).

\section{Mission concept}

In preparation for future scientific missions to the SunEarth Lagrange points, this paper considers the possibility of deploying a CubeSat from a larger spacecraft in a periodic orbit around the first (L1) or the second (L2) Lagrange points. The Sun-Earth Lagrange points are common destinations for scientific missions to observe the Sun and outer space. Over the past 40 years, seven missions have been launched to L1 (e.g., SOHO in 1995 (Domingo et al., 1995), LISA Pathfinder in 2015 (McNamara and Racca, 2009)), and four to L2 (e.g., Herschel and Planck in 2009 (Pilbratt, 2001; Tauber, 2004), Gaia in 2013 (Prusti et al., 2016)). Piggyback opportunities to L1 and L2 are certainly not as common as LEO or cislunar deployments, but a few scientific missions could provide a ride to $\mathrm{L} 2$ in the upcoming years: e.g., James Webb Space Telescope is scheduled for launch in 2019 (Greenhouse et al., 2011), and Euclid in 2020 (Laureijs et al., 2014), as well as NASA's WFIRST and ESA's PLATO and ARIEL missions which are planned for the mid- and late-2020s (ESA, 2017a, 2017b; NASA, 2015a).

In particular, Lissajous and halo orbits around the Lagrange points are commonly used periodic orbits that provide continuous observation of the Sun, and continuous communication with Earth (Howell, 2001). If deployed from a larger spacecraft already orbiting L1 or L2, the CubeSat could perform one propulsive maneuver to depart from the periodic orbit, and (if necessary) a second maneuver to flyby an asteroid passing near the Earth (see Fig. 2). The span of the mission is here limited to 150 days (from the time of departure from L1 or L2) as to confine the demanding implications of long-duration, deep-space travel (see Section 3.1), and the time 


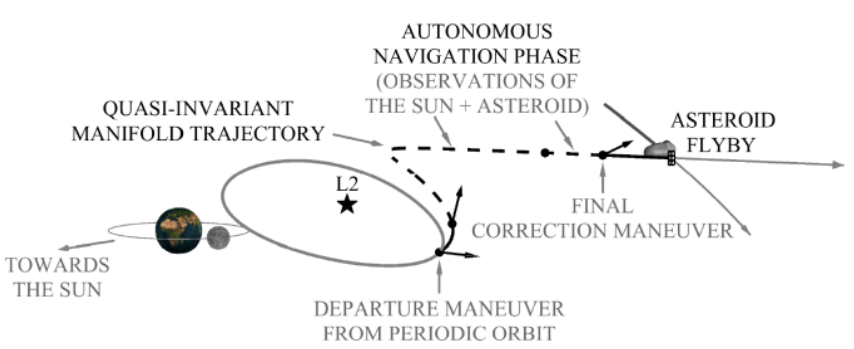

Fig. 2. Near-Earth asteroid flyby with departure from L2 halo orbit.

window considered for the mission spans from 2019 to 2025 .

As aforementioned, and in order to reduce the use of ground stations and overall cost of the mission, estimation of the CubeSat's position and velocity is performed autonomously. In this work, only observations of the Sun during cruise and observations of the asteroid prior to the flyby are used to autonomously estimate the actual states of the CubeSat as it approaches its target (for simplicity in spacecraft operations and in the navigation campaign).

The information from this navigation phase is then used to perform a final maneuver to correct the trajectory of the CubeSat and remain as close as possible to the designed flyby trajectory. This final correction maneuver is found to be essential for maximized scientific return, as otherwise the resulting trajectories would greatly differ from the designed flyby trajectories - due to, for instance, inaccuracies in the execution of the propulsive maneuvers, or to uncertainties in the position and velocity at the time of departure from around the Lagrange points.

The mission, hereafter referred to as NEARCube (Near-Earth Asteroid Reconnaissance using CubeSats), is therefore designed so that one or two (fuel-optimal) propulsive maneuvers allow a CubeSat to flyby a nearEarth asteroid from an orbit around the Sun-Earth Lagrange points. Along its <150-day trajectory, the $\mathrm{Cu}-$ beSat will also collect observations of the Sun and the asteroid for navigation purposes, and finally perform an additional correction maneuver to improve the quality of the flyby. The trajectory analysis is performed here considering the gravitational influences of the Sun and the Earth only, employing the dynamical model known as the Sun-Earth Circular Restricted Three-Body Problem (CR3BP) (Marchal, 1990; Poincaré and Magini, 1899; Szebehely and Jefferys, 1967).
Table 1

NEARCube mission requirements.

\begin{tabular}{lll}
\hline & Requirement & Description \\
\hline $\begin{array}{l}\text { 1. } \\
\begin{array}{l}\text { Sun-Earth L1/L2 } \\
\text { piggyback } \\
\text { opportunity } \\
\text { between 2019 } \\
\text { and 2025 }\end{array}\end{array}$ & $\begin{array}{l}\text { CubeSat is deployed from a } \\
\text { larger spacecraft in an orbit } \\
\text { around L1 or L2. CubeSat } \\
\text { should withstand environmental } \\
\text { conditions before and after de- } \\
\text { ployment } \\
\text { On-board propulsion system } \\
\text { should allow the CubeSat to } \\
\text { reach an asteroid from L1/L2 } \\
\text { and to correct its own trajectory } \\
\text { prior to the flyby } \\
\text { capability }\end{array}$ & $\begin{array}{l}\text { Asteroid flyby trajectory should } \\
\text { be less than 150 days as to con- } \\
\text { fine environmental implications } \\
\text { of deep-space travel }\end{array}$ \\
&
\end{tabular}

4. Autonomous navigation
(a) CubeSat should autono- mously estimate its own trajec- tory prior to the flyby, using only observations of the Sun during cruise and of the asteroid before the flyby
(b) On-board computational power should suffice to process the observations and to calculate the required trajectory correc- tion maneuver before the flyby

5. Payload allocation

6. High Technology Readiness Level (TRL)

Systems design should allocate sufficient space for the integration of a science payload to perform science during the flyby

Spacecraft components should be readily available on the market, have flight heritage, or be currently under development

General mission requirements for such an asteroid flyby mission are summarized in Table 1, which strongly drive the spacecraft component selection discussed in Section 3.

\section{State of the art and mission specifications}

CubeSats have dramatically grown in popularity since their first launch in 2003 (Swartwout, 2013). Nearly 800 CubeSats have been launched to LEO within the last 15 years (Nanosatellite \& CubeSat Database, 2017), and CubeSat technology continues to mature and provide more attractive applications. 
Table 2

CubeSat and small satellite missions of interest to the NEARCube concept study.

\begin{tabular}{|c|c|c|c|c|c|}
\hline Name & Year & Destination & Duration & Description & References \\
\hline $\mathrm{MarCO}$ & 2018 & Mars flyby & 6 months & $\begin{array}{l}\text { NASA's two 6U CubeSat mission to flyby } \\
\text { Mars for telecommunication support of In- } \\
\text { Sight mission during entry, descend and land- } \\
\text { ing (EDL) }\end{array}$ & $\begin{array}{l}\text { (Klesh and Krajewski, } \\
\text { 2015; Schoolcraft et al., } \\
\text { 2016) }\end{array}$ \\
\hline INSPIRE & 2018 & $\begin{array}{l}\text { Earth-escape } \\
\text { trajectory }\end{array}$ & 3 months & $\begin{array}{l}\text { NASA's two 3U CubeSat mission to be de- } \\
\text { ployed in an Earth-escape trajectory and } \\
\text { demonstrate deep-space telecommunications } \\
\text { and navigation capabilities }\end{array}$ & $\begin{array}{l}\text { (Klesh et al., 2013a, } \\
\text { 2013b) }\end{array}$ \\
\hline Arkyd-6 & 2018 & LEO & 2 years & $\begin{array}{l}\text { Planetary Resources' 6U CubeSat mission in } \\
\text { LEO to demonstrate water detection capabili- } \\
\text { ties on asteroids (e.g., mid-wave infrared im- } \\
\text { ager, attitude determination, power system, } \\
\text { etc.) }\end{array}$ & $\begin{array}{l}\text { (Planetary Resources, } \\
2018,2017 \text { ) }\end{array}$ \\
\hline NEA Scout & 2019 & $\begin{array}{l}\text { Asteroid } \\
\text { flyby }\end{array}$ & 2.5 years & $\begin{array}{l}\text { NASA's 6U CubeSat mission to be deployed } \\
\text { by NASA's SLS and flyby an asteroid using } \\
\text { solar sail propulsion }\end{array}$ & $\begin{array}{l}\text { (Castillo-Rogez et al., } \\
\text { 2014; Marinan et al., } \\
\text { 2017; Mcnutt et al., 2014) }\end{array}$ \\
\hline Prospector-1 & 2020 & $\begin{array}{l}\text { Asteroid } \\
\text { landing }\end{array}$ & 1.5 years & $\begin{array}{l}\text { Deep Space Industries' } 50-\mathrm{kg} \text { microsatellite to } \\
\text { land and prospect water and mineral resources } \\
\text { on an asteroid using a water-based propulsion } \\
\text { system }\end{array}$ & (Bonin et al., 2016) \\
\hline M-ARGO & 2021 & $\begin{array}{l}\text { Asteroid } \\
\text { rendezvous }\end{array}$ & 3 years & $\begin{array}{l}\text { ESA's } 12 \mathrm{U} \text { CubeSat mission study to be de- } \\
\text { ployed on a Sun-Earth L } 2 \text { transfer trajectory } \\
\text { and orbit around an asteroid }\end{array}$ & (Walker et al., 2017) \\
\hline
\end{tabular}

The recent availability of high-TRL (technology readiness level), off-the-shelf components for CubeSats allows for a significant reduction in the overall cost of building a spacecraft. As such, space agencies have shown great interest in potential CubeSat missions beyond LEO (i.e., the Moon, comets and asteroids, and other planets).

Summarized in Table 2 are CubeSat missions of interest to the NEARCube mission study, which are often referenced to along this paper. As a few examples, planned and on-going interplanetary CubeSat missions include NASA's MarCO mission to flyby Mars using two 6U CubeSats (Schoolcraft et al., 2016), NASA's INSPIRE mission to place two 3U CubeSats on an Earth-escape trajectory (Klesh et al., 2013b), and the launch of thirteen $6 \mathrm{U}$ CubeSats to the lunar vicinity and beyond along NASA's new SLS (Singer et al., 2017). Multiple other mission concepts have been proposed as well, such as flying two $3 \mathrm{U}$ CubeSats to the binary nearEarth asteroid Didymos along ESA's Asteroid Impact Mission (AIM) (Michel et al., 2016), or deploying $\mathrm{Cu}-$ beSats in the proximity of Jupiter's moon Europa along NASA's Europa Clipper mission (Goel et al., 2017; Lorenz et al., 2015).

\subsection{Current state of CubeSat technology}

Proposed and past missions define the current state of CubeSat technology, and help determine realistic subsystem specifications for the NEARCube mission:

a) Propulsion system: Few CubeSats have featured propulsion systems up to date, and with limited performance (Lemmer, 2017). Several solutions have been recently developed to meet the more demanding requirements of interplanetary CubeSats, and are now ready (or nearly ready) for use.

Deep Space Industries, for instance, offers $1.5 \mathrm{U}$ and $2.5 \mathrm{U}$ water-based thrusters providing $440 \mathrm{~N} \cdot \mathrm{s}$ and 1300 $\mathrm{N} \cdot \mathrm{s}$ of total impulse, with respective minimum impulse bits of $25 \mathrm{mN} \cdot \mathrm{s}$ and $50 \mathrm{mN} \cdot \mathrm{s}$ (Deep Space Industries, 2016). Their thrusters will be flight-tested in the 2018 Prospector-X and HawkEye 360's Pathfinder missions (Bonin et al., 2016). VACCO Industries also developed a 2U cold gas propulsion system for the $2018 \mathrm{MarCO}$ mission (25-mN nominal thrust, 755-N·s total impulse) (VACCO Industries, 2015), and now also offers a variety of cold and warm gas propulsion modules for $\mathrm{Cu}-$ beSats. Of particular interest to the NEARCube mission is their warm gas Propulsion Unit for CubeSats (PUC), 
which is scalable from $0.14 \mathrm{U}$ to $1 \mathrm{U}$, and provides high $\Delta \mathrm{V}$ capabilities (e.g., $0.5 \mathrm{U}$ in size, total impulse of 320 $\mathrm{N} \cdot \mathrm{s}$, and nominal thrust of $5 \mathrm{mN}$ ) (CU Aerospace, 2017).

b) Attitude determination and control system (ADCS): ADCS-equipped CubeSats have been extensively flown in LEO (Xia et al., 2017), and ADCS units of fully-integrated sensors and actuators are now available on the market (Blue Canyon Technologies, 2017; Hyperion Technologies B.V., 2016a; Maryland Aerospace, 2016).

As an example, the first interplanetary CubeSats will be equipped with Blue Canyon Technology (BCT)'s allin-one XACT modules. These 0.5 ADCS units were recently reported to provide $3 \sigma$ pointing accuracy and knowledge better than $\pm 0.02 \mathrm{deg}$ in LEO (along the boresight direction-cross-axis specification is 2 to 3 times better) (Mason et al., 2017). The XACT module has now been adapted for use in the deep-space MarCO and INSPIRE missions as well (Palo et al., 2013; Schoolcraft et al., 2016). The same level of pointing knowledge is expected in the INSPIRE mission, which will be provided by BCT's Nano Star Camera that is already integrated in the ADCS unit (Palo et al., 2013).

c) Communications system: high data rate $\mathrm{X}$-band transmitters (usually preferred for deep-space missions) have significant flight heritage in LEO (Nanosatellite \& CubeSat Database, 2017), and numerous 2-W, 0.1U$0.3 \mathrm{U}$ off-the-shelf options providing transmission data rates of up to 50-100 Mbps from LEO are readily available (Clyde Space, 2017; Fernandez et al., 2015).

The first interplanetary CubeSat X-band transmitters (the Iris X-band radio) were developed by JPL for the INSPIRE and MarCO missions (Duncan et al., 2014; NASA, 2015b), and will be used by NEA Scout as well (Mcnutt et al., 2014). On board MarCO CubeSats and supported by DSN 70-m antennas, these $0.5 \mathrm{U}$ transmitters (which are capable of producing a radio frequency (RF) output power of up to $5 \mathrm{~W}$ ) will be able to downlink data from Mars ( 1 AU) at $8 \mathrm{kbps}$ (using 28-dB high gain antennas, and a $4-\mathrm{W}$ transmission output) (Hodges et al., 2017). On the other hand, each INSPIRE CubeSat will be equipped with two 5-dB low gain patch antennas and will be able to transmit data at up to $1 \mathrm{kbps}$ from a distance of 0.01 AU (supported by DSN 34-m antennas, and with a 1-W transmission output) (Duncan et al., 2014; Klesh et al., 2013b).

Antenna selection will be primarily dependent on the transmission rate requirement and stowage volume constraints. Several alternatives are available in the form of patch antennas (5-10-dB gains), reflectarray antennas $(\sim 30 \mathrm{~dB})$, mesh reflector antennas $(\sim 40 \mathrm{~dB})$, etc. (Lokman et al., 2017). A noteworthy solution are MarCO's reflectarray antennas (Hodges et al., 2017), whose low stowage volume $(\sim 0.1 \mathrm{U})$ and high gain $(\sim 28$ $\mathrm{dB})$ represent an attractive solution for future deepspace CubeSat missions.

d) Electrical power system (EPS): power generation on small spacecraft is generally accomplished with solar cells (NASA, 2015c). Current solar cell efficiencies range from $26 \%$ to $33 \%$, and off-the-shelf components can be found on various configurations. Surfacemounted panels, for instance, provide power levels of the order of $10 \mathrm{~W}$ in LEO (DHV Technology, 2015; EnduroSat, 2016). Deployable panels, however, can currently provide up to $30-35 \mathrm{~W}$ thanks to their augmented surface area and (limited) adaptability of their orientation (Clyde Space, 2016a; MMA Design LLC, 2014).

The INSPIRE mission will be equipped with Pumpkin, Inc.'s 3Ux1U deployable solar panels that generate up to $20 \mathrm{~W}$ (at $1 \mathrm{AU}$ from the Sun) (Klesh et al., 2013b), whereas MarCO CubeSats will generate up to $35 \mathrm{~W}$ using two 2Ux3U solar panels that unfold from the $3 \mathrm{Ux} 1 \mathrm{U}$ sides of the spacecraft (developed by MMA Design LLC) (Klesh and Krajewski, 2015).

The use of batteries as the primary source of power is not common due to their limited lifespan (up to a week). Rechargeable lithium ion and lithium polymer secondary batteries, nevertheless, are extensively used for power storage in small spacecraft (NASA, 2015c). These batteries provide high energy-to-weight ratios (150-250 W'h $\left.\cdot \mathrm{kg}^{-1}\right)$ (Navarathinam et al., 2011), and are indispensable for times when not sufficient solar energy is available. Both, MarCO and INSPIRE missions, will be equipped with rechargeable batteries (Klesh et al., 2013b; Schoolcraft et al., 2016). The MarCO mission, for instance, will use a set of twelve $18 \mathrm{~mm}$ x $65 \mathrm{~mm}$ lithium ion cells, which amount for approximately $0.25 \mathrm{U}$. The INSPIRE mission will use a pack of four battery cells instead $(0.2 \mathrm{U})$, which were inherited from the RAX and RAX-2 LEO missions (Springmann et al., 2012; The University of Michigan, 2009).

In addition to the power generation and storage components, power management and distribution (PMAD) on CubeSats is achieved through the EPS motherboard, which also protects electronics and batteries throughout the spacecraft (NASA, 2015c). As an example, Clyde Space and GomSpace provide single-board off-the-shelf PMAD solutions $(0.15 \mathrm{U})$ that feature battery charge regulators, regulated power buses at different voltages (e.g., $3.3 \mathrm{~V}, 5 \mathrm{~V}, 12 \mathrm{~V})$, and can also be integrated with $0.2 \mathrm{U}$ 
battery packs (Clyde Space, 2016b; GomSpace, 2014). The INSPIRE and MarCO missions both used 0.2U PMAD boards with heritage from the RAX and RAX-2 LEO missions (Klesh and Krajewski, 2015; Klesh et al., $2013 \mathrm{~b}$ ), with regulated $3.3-\mathrm{V}$ and $5-\mathrm{V}$ power buses (MarCO also included a 12-V battery bus).

e) Science payload: miniaturization of scientific instruments for CubeSats is an ongoing effort in industry, space agencies and academia. As a result, a variety of scientific instruments (including off-the-shelf components) could now be allocated within $1 \mathrm{U}$ or less.

For example, 0.3U-1U visible cameras have been greatly developed for Earth observation purposes, and ground resolutions of $600 \mathrm{~m}$ down to $30 \mathrm{~m}$ per pixel are now possible from LEO ( $650 \mathrm{~km}$ altitude) with off-theshelf solutions (Crystalspace, 2015; GomSpace, 2017; SCS Space, 2017). Ultraviolet (UV), visible (VIS), and infrared (IR) spectrometers in the 190-2400-nm bandpass and $0.1-10-\mathrm{nm}$ optical resolutions are also available in <0.2U modules (Ocean Optics, 2016, 2013a; Thoth Technology, 2013). Far-UV and mid-IR spectrometers in the 90-200-nm and 3000-8000-nm ranges are also under development (Farrah et al., 2017; NASA, 2017). Ion and neutral mass spectrometers able to resolve $\mathrm{H}, \mathrm{He}, \mathrm{N}, \mathrm{N}_{2}, \mathrm{O}$ and $\mathrm{O}_{2}$, and $0.7 \mathrm{U}-1.3 \mathrm{U}$ in size, were designed for missions ExoCube and Dellingr, and constellation mission QB50 (Chaudery, 2014; Johnson et al., 2015), and 60-200-GHz microwave spectrometers (1U in size) were also developed for missions MicroMAS, MiRaTA and TROPICS (Blackwell, 2015; Cahoy et al., 2017). High-resolution ( 0.1 nT) fluxgate magnetometers for scientific use have been flown as well, including the boom-mounted, minimal-intrusion (approximately $0.1 \mathrm{U}$ ) magnetometer employed in the QB50 project (Miles et al., 2016).

In conclusion, there exists a number of flight-ready scientific instruments, and their selection should be made in accordance with the volume constraints and scientific objectives of the mission. This selection process should also consider flyby characteristics (e.g., flyby altitude, relative velocity, etc.) and existing instrument capabilities, as these will determine attainable scientific goals for the mission.

f) Environmental considerations: environmental factors to consider when designing an interplanetary CubeSat mission include radiation effects and thermal control.

Thermal control technology has extensive flight heritage from traditional missions (Gilmore, 2002), and is generally achieved through multi-layer insulation (MLI) blankets, thermal coating (paint or tape), thermal straps, louvers, deployable radiators, heaters, etc. Thermal management in small spacecraft becomes more challenging due to volume and power constraints, especially far away from the Sun. Previous CubeSat missions have successfully flown using MLI and coating techniques (NASA, 2015c), and further development and validation of thermal control systems for small spacecraft is an ongoing effort in the community (Kang and Oh, 2016; Katke et al., 2016; Wachche et al., 2014).

Typical operating temperatures for the components discussed in the current section range between $-15^{\circ} \mathrm{C}$ and $+50{ }^{\circ} \mathrm{C}$ : e.g., thrusters (CU Aerospace, 2014), star trackers (Hyperion Technologies B.V., 2016b; Palo et al., 2013), radio transmitters (Clyde Space, 2017; NASA, 2015b), or ADCS units (Hyperion Technologies B.V., 2016a; Maryland Aerospace, 2016). Microprocessors and batteries, for instance, can operate in even wider temperature ranges: e.g., between $-40{ }^{\circ} \mathrm{C}$ and +120 ${ }^{\circ} \mathrm{C}$ (Cubic Aerospace, 2017; EXA, 2016; Hannu Leppinen, Antti Kestilä, Pauli Pihajoki, Jukka Jokelainen, 2014). Whereas some science payloads (e.g., visual imagers, spectrometers) might introduce more demanding temperature constraints: e.g., operating temperatures above $0-10^{\circ} \mathrm{C}$ and below $+30-40^{\circ} \mathrm{C}$ (SCS Space, 2017; Thoth Technology, 2013).

Mars-bound 6U MarCO CubeSats, for instance, will use blankets, radiators, and heaters to achieve thermal balance in a strongly varying thermal environment (Klesh and Krajewski, 2015); whereas thermal considerations for the INSPIRE and NEA Scout missions represent less of a challenge since they will remain at $\sim 1$ AU from the Sun (Klesh et al., 2013b; Mcnutt et al., 2014). Proper heat dissipation from strong emitting sources, however, is essential for such missions (e.g., thrusters, communications system, batteries). Similarly to the NEARCube mission, these missions are continuously exposed to sunlight (solar flux is $\sim 1370 \mathrm{~W} / \mathrm{m}^{2}$ at 1 AU) and only passive thermal systems are generally available. Deployable radiators with low stowage volume, for instance, could greatly benefit such missions (Bunce et al., 2016). However, volume constraints are often too stringent, hence thermal mitigation should be accomplished through thermal coating techniques (Escobar et al., 2016), in conjunction with careful duty cycling of strongly emitting components to avoid overheating (which is compatible with NEARCube's simple concept of operations). 
An additional challenge for the NEARCube spacecraft will be maintaining temperature of its critical components (batteries cells, electronic boards, payload) during the piggyback phase. Although further analysis of the thermal requirements and environmental conditions throughout the mission is necessary, the MASCOT lander (10-kg asteroid lander aboard JAXA's Hayabusa2 spacecraft) provides a valuable example of a successful thermal design for secondary payloads (Celotti et al., 2015; German Aerospace Center, 2016). Launched in 2014, the Hayabusa-2 spacecraft carried the MASCOT lander during its 3.5-year-long cruise phase to asteroid (162173) Ryugu. Thermal balance was achieved primarily through passive systems (i.e., painting, coatings, heat pipes, MLI, interface tuning, and a radiator facing external conditions). Limited heating power was also supplied to heaters by the Hayabusa-2 spacecraft during cold phases of its cruise to maintain proper temperature of the electronic box, primary battery cells, and payload. If possible and necessary, health checks or maintenance activities could also be performed through an umbilical connector to the main spacecraft (German Aerospace Center, 2016; Innovative Solutions in Space, 2013).

Similarly, radiation effects become an important consideration for long-span missions and when flying through high-radiation environments (such as the Van Allen belt or solar particle events) (Bourdarie and Xapsos, 2008). Mitigation techniques often imply shielding sensitive individual components, whole sections or the entirety of the spacecraft, or sheltering critical components within the spacecraft with less sensitive components (Maurer et al., 2008). Watchdog architectures are also a common solution (which generally require radiation hardening as well): these are independent systems that monitor the state of a processing unit, and refresh or reset the system to a previously working state in the event of a software fault (e.g., due to a SingleEvent Upsets (SEUs)). Protection against frequent SEUs in deep-space travel is essential, and therefore watchdog strategies should be implemented regardless of the extent of the mission (e.g., INSPIRE, MarCO and NEA Scout missions will use these (Klesh et al., 2013b; Mcnutt et al., 2014; Schoolcraft et al., 2016)). Additional shielding is also required for long-duration missions that will be exposed to large Total Ionizing Dose (TID) levels. Radiation effects represent an active field of research in the community, and solutions for small satellites in deep-space travel (e.g., NEA Scout mission) have already been proposed (Kahn et al., 2017).

Several of the components discussed in the current section have either proven year-long lifetimes in LEO- where the Van Allen belt also induces high levels of radiation-(e.g., >3-year BCT's XACT unit (Hegel, 2016), >30-year star trackers (Jørgensen et al., 2005), $>4$-year batteries (EXA, 2016)), have been radiationhardened (e.g., watchdog units and standalone on-board computers with $>300$-krad radiation tolerances and $10^{-15}$ SEUs per day (VORAGO Technologies, 2018)), or have been specifically developed for deep-space environment (e.g., JPL's Iris radio (Duncan et al., 2014)). Some components, however, will still require radiation-hardened watchdog architectures to detect and correct SEUs (e.g., on-board computer, star tracker, sun sensor, science payload, etc.), or further aluminum shielding to reduce SEU frequencies and TID levels (e.g., science payload, or if components with lower radiation tolerances-i.e., 10-50 krad - are selected (Hyperion Technologies B.V., 2016b; SCS Space, 2017)). For reference, analyses for the LISA Pathfinder and JWST missions show that typical radiation doses (with minimal or no shielding) in the Sun-Earth L1/L2 regions are 100 krad per year (Barth et al., 2000; EADS Astrium Ltd, 2005), and similar environmental conditions can be expected on NEARCube's asteroid flyby trajectory. According to these two mission studies, 10-20-krad/year radiation levels can be achieved with reasonable $0.5-1-\mathrm{mm}$ aluminum shield thicknesses, which should suffice to protect the most sensitive components during the <150-day NEARCube mission.

g) Other considerations: various other noteworthy considerations are associated to low-cost, small spacecraft missions. In the context of a stringent budget for the mission, some of these include the design of ground data systems, and spacecraft reliability aspects.

Ground operations for CubeSat missions have also seen a shift towards cost reduction, with solutions such as employing a single ground antenna operated by a single computer, or communicating with collaborative networks of volunteering amateur radio operators (Klofas, 2006). CubeSats have commonly operated in amateur, low radio frequencies (e.g., VHF and UHF bands) (Klofas and Leveque, 2012), but the higher performance of recent missions and increased volume of valuable data are promoting the use of more sophisticated (and costly) networks provided by companies and governmental organizations (Cheung et al., 2016). These networks also display a wide range of capabilities in terms of supported frequency bands (including $\mathrm{X}$ - and Kaband) and antenna sizes (3- to 70-m antennas), with some moderate solutions at less than $\$ 500$ per pass (ATLAS Space Operations, 2017; SpaceNews, 2017a), and governmental networks such as NASA's DSN (34- 
and 70-m antennas) or ESA's ESTRACK (15- and 35$\mathrm{m}$ antennas) at the higher end of the spectrum (at hourly rates of thousands of USD\$). Therefore, careful consideration of necessary ground station capabilities and services is required, as they can also drastically increase the cost of a mission.

Although long-duration missions should not be discouraged, they are especially susceptible to communications, on-board computer, and power system failures (Langer and Bouwmeester, 2016). In fact, pico- and nano-satellites (which include 3U and 6U CubeSats) display particularly steep decrements in their mission success rates with time (Guo et al., 2014). Conversely, missions that have been designed for longer lifetimes often show more optimistic success rates, as these usually entail larger resources and more rigorous design processes than short-duration missions (Guo et al., 2014). Hence, special consideration of mission duration, design robustness, and cost is also necessary.

\subsection{NEARCube mission specifications}

As aforementioned, several interplanetary CubeSat missions have already been proposed and planned, including the NEA Scout $6 \mathrm{U},<12-\mathrm{kg}$ CubeSat mission to flyby an asteroid using primarily off-the-shelf components and solar sail propulsion (Mcnutt et al., 2014). This mission concept has been accepted for launch as secondary payload on NASA's SLS, and is expected to achieve a $<10-\mathrm{km}, 10-\mathrm{m} / \mathrm{s}$ slow flyby of a $<50-\mathrm{m}$ asteroid within its 2.5-year lifespan (Castillo-Rogez et al., 2014). High-resolution imaging data $(10 \mathrm{~cm} /$ pixel $)$ will be transmitted at a distance of $<1$ AU from Earth at $\geq 1$ kbps using NASA's DSN 34-m antennas (Marinan et al., 2017).

In contrast to the NEA Scout 6U mission, the current work explores the possibility of flying by an asteroid using an autonomous $3 \mathrm{U}$ CubeSat $(<4 \mathrm{~kg})$ : in an effort to demonstrate that near-Earth asteroid exploration is possible with even smaller spacecraft, with reduced use of large, costly ground stations, and with readily-available components. In specific, short-duration missions $(<150$ days) are considered in order to-as outlined in Section 3.1-limit thermal and radiation concerns of deep-space travel, reduce the demand on the power and communications systems and on ground antennas, and to confine the reliability issues of long-duration missions.

Such a mission, however, imposes a high demand on some other subsystems and drives the preliminary design of the NEARCube spacecraft. The propulsion system, for instance, should provide sufficient $\Delta \mathrm{V}$ to travel
Table 3

NEARCube 3U spacecraft model.

\begin{tabular}{|c|c|c|}
\hline Parameter & $\begin{array}{l}\text { Used } \\
\text { value }\end{array}$ & Reference \\
\hline Total mass & $4 \mathrm{~kg}$ & $\begin{array}{l}\text { 3U CubeSat design speci- } \\
\text { fication (Mehrparvar, } \\
\text { 2014) }\end{array}$ \\
\hline Total $\Delta \mathrm{V}$ & $80 \mathrm{~m} / \mathrm{s}$ & $\begin{array}{l}\text { VACCO's 0.5U PUC, 85- } \\
\text { m/s } \Delta V \text { (CU Aerospace, } \\
2017)\end{array}$ \\
\hline $\begin{array}{l}\Delta \mathrm{V} \text { magnitude } \\
\text { accuracy }(3 \sigma)\end{array}$ & $1 \%$ & $\begin{array}{l}\text { VACCO's } 0.25 U \text { PUC, } \\
<5 \% \text { magnitude uncer- } \\
\text { tainty (CU Aerospace, } \\
2017)^{\mathrm{a}}\end{array}$ \\
\hline $\begin{array}{l}\text { ADCS pointing } \\
\text { accuracy }(3 \sigma)\end{array}$ & $\pm 0.02 \mathrm{deg}$ & $\begin{array}{l}\text { BCT's } 0.5 \mathrm{U} \text { XACT, } \\
\pm 0.02 \text {-deg boresight } \\
\text { pointing accuracy (Mason } \\
\text { et al., 2017) }\end{array}$ \\
\hline $\begin{array}{l}\text { ADCS pointing } \\
\text { knowledge }(3 \sigma)\end{array}$ & $\pm 0.01 \mathrm{deg}$ & $\begin{array}{l}\text { PROBA-2's star tracker, } \\
\pm 0.0006-\text { deg pointing } \\
\text { knowledge (Jørgensen et } \\
\text { al., 2005) }\end{array}$ \\
\hline $\begin{array}{l}\text { RF output } \\
\text { power }\end{array}$ & $<5 \mathrm{~W}$ & $\begin{array}{l}\text { JPL's } 0.5 \mathrm{U} \text { Iris X-band } \\
\text { radio, } 5 \text {-W RF output } \\
\text { power (NASA, 2015b) }\end{array}$ \\
\hline Antenna gain & $5 \mathrm{~dB}$ & $\begin{array}{l}\text { INSPIRE's low gain } \\
\text { patch antennas, } 5 \text {-dB peak } \\
\text { gain (Duncan et al., 2014) }\end{array}$ \\
\hline $\begin{array}{l}\text { Generated } \\
\text { power }\end{array}$ & $35 \mathrm{~W}$ & $\begin{array}{l}\text { MMA Design's HaWK }{ }^{\mathrm{TM}} \text {, } \\
\text { 36-W peak power (MMA } \\
\text { Design LLC, 2014) }\end{array}$ \\
\hline
\end{tabular}

${ }^{a}$ Thrust uncertainty below $5 \%$ includes experimental measurement error.

${ }^{\mathrm{b}}$ Cross-axis pointing accuracy of BCT's XACT is \pm 0.006 deg (Mason et al., 2017), better than boresight pointing accuracy, and also below used value.

${ }^{c}$ Cross-axis pointing knowledge of the star tracker originally integrated in BCT's XACT is \pm 0.006 deg (Palo et al., 2013), also below used value.

to an asteroid within the limited duration of the mission. The capabilities of the ADCS system will determine the directional accuracy of the propulsive maneuvers as well as the quality of the observations for navigation purposes, which consequently translates into closer flybys and higher scientific return. And although the power and communications systems are not required to operate at far distances from the Earth and Sun, they must provide adequate performance throughout the mission while allocating sufficient space for the science payload. 
The proposed NEARCube spacecraft design is summarized in Table 3, which assumes component performance in accordance to the state of the art discussed in Section 3.1. The NEARCube spacecraft is thus designed with JPL's 0.5U Iris X-band radio, MMA Design's 2Ux3U HaWK $^{\mathrm{TM}}$ solar array ( 0.1U intrusion), VACCO's 0.5U PUC propulsion unit, INSPIRE's 5-dB patch antennas, and BCT's 0.5U XACT ADCS module (implemented with PROBA-2's star tracker instead (Jørgensen et al., 2005), for higher optical sensitivity than its current star tracker to detect the target asteroid sufficiently early - further discussion on this selection is provided in Section 5).

It is worth mentioning that no particularly stringent telecommunication requirements are anticipated for a mission such as NEARCube: autonomous navigation and its simple mission profile will reduce telecommunication needs to the downlink of science data and to seldom transmission of telemetry and commands (here it is also assumed that most spacecraft operations can be automated through time-tagged commands). Additionally, BCT's ADCS unit can also provide high pointing accuracies that would result in reasonable pointing losses, and science data, for instance, could be downlinked following the flyby with no particular time restrictions either. Although further analysis is still required (e.g., ground antenna selection, desired data rates, etc.), a simple solution is proposed in this study: 5-dB patch antennas, in line with the design of the INSPIRE mission and although more sophisticated solutions could be implemented (e.g., MarCO's 28-dB reflectarray antennas).

Such a design would allow for approximately $1.5 \mathrm{U}$ for the command and data handling $(\mathrm{C} \& \mathrm{DH})$ system, the EPS module, and the science payload. Similarly to the INSPIRE and MarCO missions, for instance, the design could include two MSP430 microprocessors in stack (0.3U) (Schoolcraft et al., 2016). INSPIRE will specifically use a single microprocessor that provides basic command and data handling, a real-time-clock, interfaces with other systems, monitors spacecraft health, and includes a watchdog architecture (Klesh et al., 2013b). The second board can then be used to process data from the science payload. Such a design could be based on the high-performance, flight-tested TMS570 microchips, which include a dual-processor architecture for error identification, and have been previously used in CubeSats (Hannu Leppinen, Antti Kestilä, Pauli Pihajoki, Jukka Jokelainen, 2014; Taylor et al., 2011). Alternatively, graphics processing units (GPUs) and field-programmable gate arrays (FPGAs) could also provide a solution for high-performance on-board data processing (Arnold et al., 2012; Bekker et al., 2010; Buonaiuto et al., 2017; Cubic Aerospace, 2017).

The EPS module could be composed of a four-cell battery pack like the one used by INSPIRE $(0.2 \mathrm{U})$, and by a single EPS motherboard such as the ones provided by Clyde Space and GomSpace (0.15U), or the EPS boards used by INSPIRE and MarCO (0.2U).

This design would effectively allocate approximately $0.8 \mathrm{U}$ for the science payload (without explicit redundancy of any components). As outlined in Section 3.1, several instruments could be allocated within $1 \mathrm{U}$ or less, such as a camera, remote sensing instruments (e.g., UVVIS-IR spectrometers, mass spectrometers), or boommounted magnetometers. However, this selection will be dependent on the attainable flyby altitudes and on the specific scientific objectives of the mission, which are further discussed in Sections 5 and 6, respectively.

\section{Target selection and trajectory design}

Our target selection process begins by understanding how far a CubeSat could travel from its departure periodic orbit around L1 or L2. In specific, this work utilizes for reference in size the Lissajous periodic orbits used by LISA Pathfinder (launched in 2015 to L1 (McNamara and Racca, 2009)) and James Webb Space Telescope (scheduled for launch in 2019 (Space Telescope Science Institute, 2004)). The departure periodic orbits are both modeled in the CR3BP as halo orbits instead (Howell, 1984; Howell and Pernicka, 1987)—as frequently done for mission planning to the Lagrange points (Barden et al., 1996). The departure orbits considered here are an L1 Northern halo and an L2 Southern halo, both $\sim 500,000 \mathrm{~km} \times 1,500,000 \mathrm{~km} \times 800,000 \mathrm{~km}$ in size.

Lissajous and halo orbits are unstable orbits and require station-keeping maneuvers for a spacecraft to remain in them (Goudas, 1963). Stability analysis of these orbits shows that, at any point along the periodic orbit, there exists a most favorable direction (defined in both senses) for a spacecraft to depart from it (i.e., unstable direction), and a most favorable direction for a spacecraft to be inserted into the periodic orbit (i.e., stable directions) (Simó, 1990). If a small propulsive maneuver is exerted along the unstable direction, a spacecraft would then naturally drift away from the periodic orbit at virtually no cost. The set of all trajectories departing in the unstable direction from the periodic orbit is known as the "unstable invariant manifold" of that particular orbit. 
Extensive literature is available on the exploitation of invariant manifolds for mission design purposes, especially for the design of low- $\Delta \mathrm{V}$ transfer trajectories to or between periodic orbits around the Lagrange points (Barden et al., 1996; Gómez et al., 1993; Gómez and Masdemont, 2000; Howell et al., 1994). Provided the very limited $\Delta \mathrm{V}$ capability of CubeSats, departure trajectories from the periodic orbit are found to remain close to those trajectories on the unstable invariant manifold. Therefore, the unstable invariant manifold is used in this study as a first approximation of the region that can be reached by the CubeSat.

As previously mentioned, the unstable direction for departure is defined in both senses: one of them produces trajectories towards the Earth, and the opposite sense produces trajectories away from the Earth. Earth-bound trajectories would generally cause a CubeSat to get trapped by Earth's gravitational field and not allow it to reach far destinations within the limited mission span (150 days). Therefore, only departure trajectories that follow the invariant manifold in the opposite direction to the Earth are considered here, as these will allow the CubeSat to naturally travel long distances and potentially intersect the trajectory of a near-Earth asteroid.

The first step in the target selection process is, consequently, to approximate the CubeSat's reachable region by generating trajectories on the unstable invariant manifold of the departure halo orbits (away from Earth). Taking into account the maximum mission span (150 days) and maximum available $\Delta \mathrm{V}(80 \mathrm{~m} / \mathrm{s})$, the invariant manifold is approximated by introducing an $80-\mathrm{m} / \mathrm{s}$ perturbation in velocity along the unstable direction and propagating the resulting trajectories over 150 days.

The second step on the target selection process is to identify asteroids that could potentially be encountered by the CubeSat. Resulting flyby altitudes are expected to be large due to the limited capabilities of the CubeSat; therefore, the target asteroids should be sufficiently large to allow proper observations during the flyby and any significant scientific return. Through JPL's Center for Near Earth Object Studies website (NASA, 2008), asteroids larger than $\sim 100$ meters passing near the Earth are identified (i.e., absolute magnitude smaller than 22.5 and Earth flyby within $0.1 \mathrm{AU}$ ). In this work, only asteroids with Earth flybys between years 2019 and 2025 are considered, although the methodology could be directly applied to other time windows. A total of $\sim 370$ asteroids fulfilling the previous characteristics were identified. Their ephemeris data was automatedly obtained using JPL's HORIZONS telnet interface (NASA, 2013), and expressed with respect to the Sun-Earth synodic reference frame used in the CR3BP analysis.

The search for potential targets is then further restricted to asteroids whose trajectories pass close to the unstable invariant manifolds previously generated. Illustrated in Fig. 3 (represented in the Sun-Earth synodic reference frame, centered on the Earth, and projected on the ecliptic) are the initial L1 and L2 halo orbits, approximated unstable invariant manifolds, and 40 different asteroids whose trajectories pass within $0.01 \mathrm{AU}$ of the reachable regions (sized according to their estimated diameters, color-coded according to their minimum distance to the invariant manifolds, and with arrows proportional to the relative velocity with respect to the point of minimum distance on the invariant manifold).

Once those potential targets are identified, the third step on the target selection process is to design trajectories to encounter those asteroids, using either one or two propulsive maneuvers, as necessary. Considering the limited $\Delta \mathrm{V}$ of the mission, however, encountering those asteroids is not always possible.

The propulsive maneuvers are modeled in this study as instantaneous, impulsive maneuvers. It is worth mentioning, however, that the $0.5 \mathrm{U}$ thruster considered for the spacecraft has a nominal thrust of $5 \mathrm{mN}$ and a maximum continuous thrust time of $20 \mathrm{~min}$ (CU Aerospace, 2014). Furthermore, additional $20 \mathrm{~min}$ are required between burns to allow the thruster to cool down (D. Carroll at CU Aerospace, personal communication, April $23,2018)$. Provided the limited $\Delta \mathrm{V}$ that can be generated by each 20 -min burn, the total time required for the thruster to produce the available $80-\mathrm{m} / \mathrm{s} \Delta \mathrm{V}$ is approximately 35 hours. Even though this constraint imposes non-impulsive, long-duration maneuvers, the impulsivemaneuver model used here to design trajectories still holds as a valid approach. Although this analysis is not described in this paper, equivalent trajectories (i.e., $\Delta \mathrm{V}$ requirements and times of flight) can be obtained using a 20-min-on, 20-min-off continuous thrust model.

The trajectory design process is approached here as an optimization problem in which to determine: (1) the magnitude and direction of the departure impulsive maneuver, and (2) the magnitude, direction, and time after departure at which the (optional) second impulsive maneuver is executed (a total of seven variables). The objective of this optimization problem is to minimize the total $\Delta \mathrm{V}$ required to intersect the trajectory of the asteroid within the specified span of the mission, for a given departure point along the periodic orbit. 


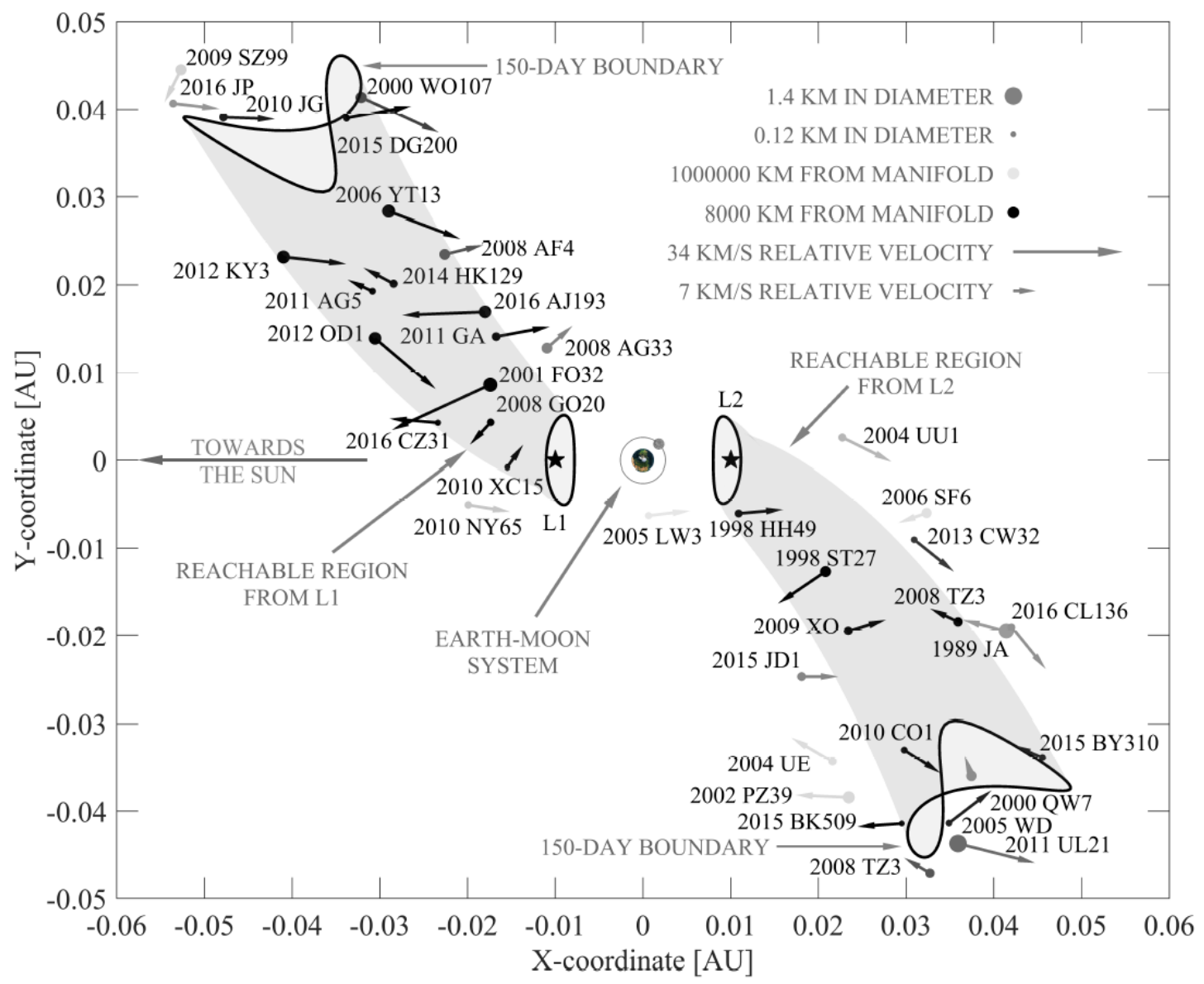

Fig. 3. Near-Earth asteroids passing close to reachable regions from L1 and L2 in the 2019-2025 time window.

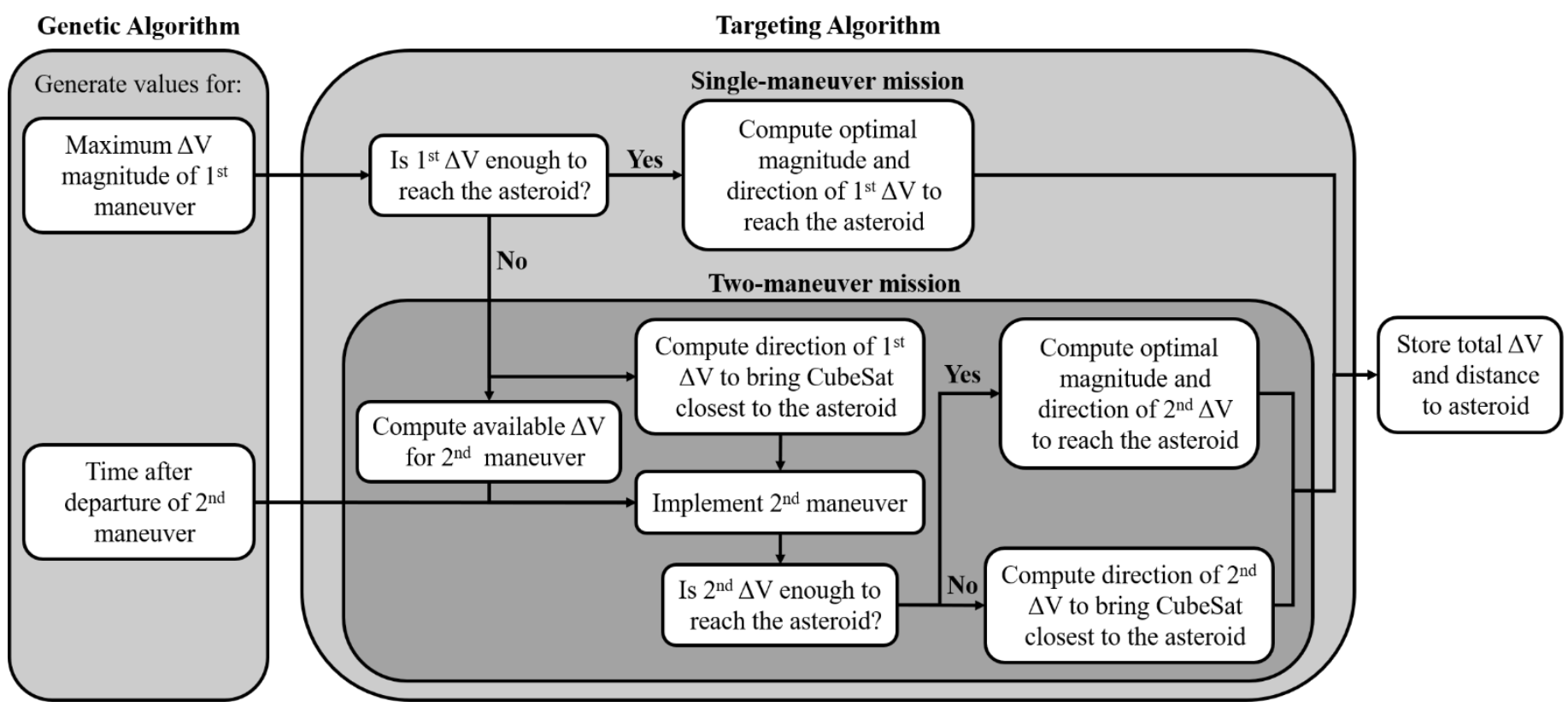

Fig. 4. Diagram of logic employed by trajectory design algorithm. 
In addition, if only one maneuver allows the CubeSat to flyby the asteroid at a lower cost than two maneuvers, then a single-maneuver mission is implemented.

A similar trajectory design problem was faced by China National Space Administration (CNSA) at the end of Chang'e-2's primary mission around the second SunEarth Lagrange point (Gao, 2013). In an effort to extend the flight of the Chang'e-2 mission, fuel-optimal trajectories to flyby a near-Earth asteroid were designed using sequential quadratic programming (SQP). The SQP algorithm determined a total of nine variables in a fourbody dynamical model (with gravitational influences of the Sun, Earth and the Moon): time of departure, time of the second maneuver, time of the flyby, and the components of each impulsive maneuver.

The optimization problem is solved here using a genetic algorithm instead-based on the open-source NSGA-II tool (Deb et al., 2002) - in combination with a differential corrector targeting algorithm (Koon et al., 2011). The genetic algorithm is employed to determine two quantities: (1) the maximum $\Delta \mathrm{V}$ magnitude that can be used for the first maneuver (the remaining $\Delta \mathrm{V}$ becomes available for the second maneuver), and (2) the time after departure at which the (optional) second maneuver is executed.

These values are then inputted into the targeting algorithm, which - through a single shooting method - efficiently optimizes the required $\Delta \mathrm{V}$ to reach the asteroid (magnitude and direction). If the $\Delta \mathrm{V}$ magnitude available for a maneuver is large enough for the CubeSat to reach the asteroid, then the targeting algorithm specifies the optimal magnitude and the direction of that maneuver. Otherwise (if the $\Delta \mathrm{V}$ is not large enough to reach the asteroid), then the available $\Delta \mathrm{V}$ is used in its entirety, and the targeting algorithm calculates the required direction to bring the CubeSat closest to the asteroid. This logic is used for each individual in the population of the genetic algorithm, and it is illustrated in Fig. 4.

For maximized scientific return and as illustrated in Fig. 5, trajectories are designed to flyby the asteroid on its illuminated face. This strategy should reduce the risk of accidentally colliding with the asteroid and allow for proper visibility during the flyby. The targeting algorithm specifically aims at a point $1000 \mathrm{~km}$ away from the asteroid in the direction of the Sun. The value of $1000 \mathrm{~km}$ is used in this study as a baseline scenario for all the asteroids under consideration, to understand the accuracy of the flybys that can be achieved (discussed in Section 5). In a real mission, this value could be ad-

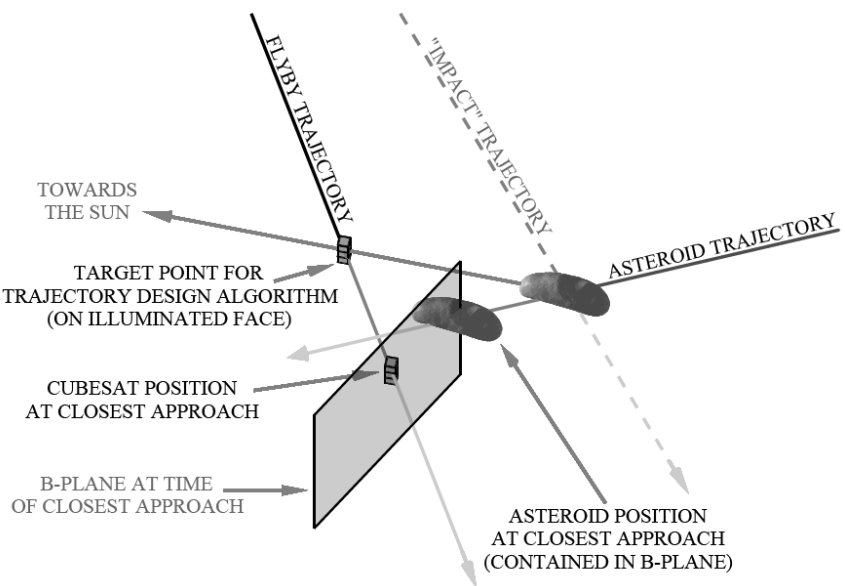

Fig. 5. Diagram of designed flyby trajectories on illuminated face of the asteroid.

justed according to the target asteroid, to the flyby accuracy that can be achieved for that particular asteroid, and to other mission requirements.

The B-plane-also shown in Fig. 5-is commonly used to illustrate flyby accuracies (Sergeyevsky et al., 1983), and is further employed to support the sensitivity analysis discussion in Section 5. The B-plane contains the position of the asteroid at the time of closest approach, and is defined as normal to the relative velocity between the asteroid and the CubeSat (on its nominal, unperturbed trajectory). The actual position of the $\mathrm{Cu}$ beSat will differ from that on the designed trajectory primarily due to inaccuracies in the execution of the maneuvers, and to uncertainties in the departure conditions (i.e., initial position and velocity). Projecting the actual position of the CubeSat onto the B-plane provides a measure to discuss the accuracy of flybys and achievable flyby altitudes for the mission.

The trajectory design process described thus far assumes the departure point from the periodic orbit is given. This departure point will generally not be chosen by design and will come predetermined by the proper timing to encounter the asteroid. In order to provide some insight on the $\Delta \mathrm{V}$ requirements as a function of the departure point, minimum $-\Delta \mathrm{V}$ trajectories are designed from nine departure points along the periodic orbits (equally spaced in time throughout one orbit period). As an example, and projected on the ecliptic, Fig. 6 illustrates the optimal $\Delta \mathrm{V}$ requirements along the departure L1 halo orbit, and corresponding time of flight (TOF) to encounter asteroid 2001 FO32 (close Earth encounter on March 21, 2021 - black dot if reachable within 150 days and with $80 \mathrm{~m} / \mathrm{s}$ of $\Delta \mathrm{V}$, grey dot otherwise). 


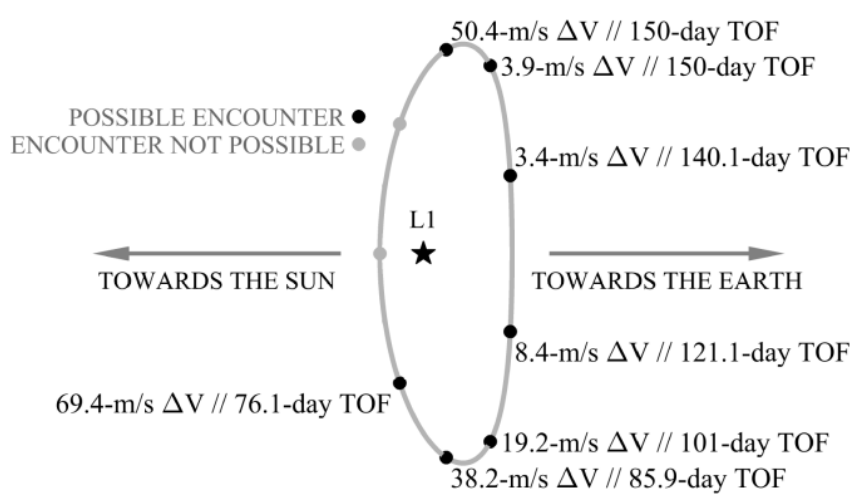

Fig. 6. $\triangle \mathrm{V}$ and TOF requirements along $\mathrm{L} 1$ halo orbit for asteroid $2001 \mathrm{FO} 32$.

From this trajectory design analysis, a total of 24 different asteroids are identified that can be reached by a CubeSat within 150 days and with $80 \mathrm{~m} / \mathrm{s}$ of total $\Delta \mathrm{V}$ (additional details on these encounters are provided in Table 4).

Finally, the fourth and last step in the target selection process is to identify asteroids that can not only be reached, but also detected by the on-board navigation camera (in our case, the on-board star tracker) prior to the encounter. As described in Section 2, observations of the asteroid can also be used to autonomously estimate the position and velocity of the CubeSat as it approaches its target. Therefore, only those asteroids that can be detected by the CubeSat sufficiently early are selected here as potential destinations (further discussion on the importance of collecting enough observations of the asteroid can be found in Section 5).

In this analysis, detectability is assessed in terms of the apparent visual magnitude of the asteroids as observed from the CubeSat. This magnitude is computed in terms of the distance between the CubeSat and the asteroid, and of their positions with respect to the Sun (Bowell et al., 1988; Lagerkvist and Magnusson, 1990). This work assumes a slope parameter of 0.15 for all asteroids-as commonly done (Dymock, 2007) — given that no data is available in this respect for the asteroids under consideration. Asteroids can be detected by the CubeSat only if their apparent visual magnitude is below a certain detectability threshold. The value of this threshold is dependent on the capabilities of the navigation camera (i.e., its sensitivity), and a value of 15 is assumed in this study. Such sensitivity can be accomplished, for example, by one of the four cross-strapped $5 \mathrm{~cm} \times 5 \mathrm{~cm} \times 5$ $\mathrm{cm}(0.125 \mathrm{U})$ camera head units (CHUs) composing the star tracker used by ESA's PROBA-2 mission (Jørgensen et al., 2005). The higher power consumption of these CHUs $(\sim 3.4 \mathrm{~W})$ allows for an improvement in

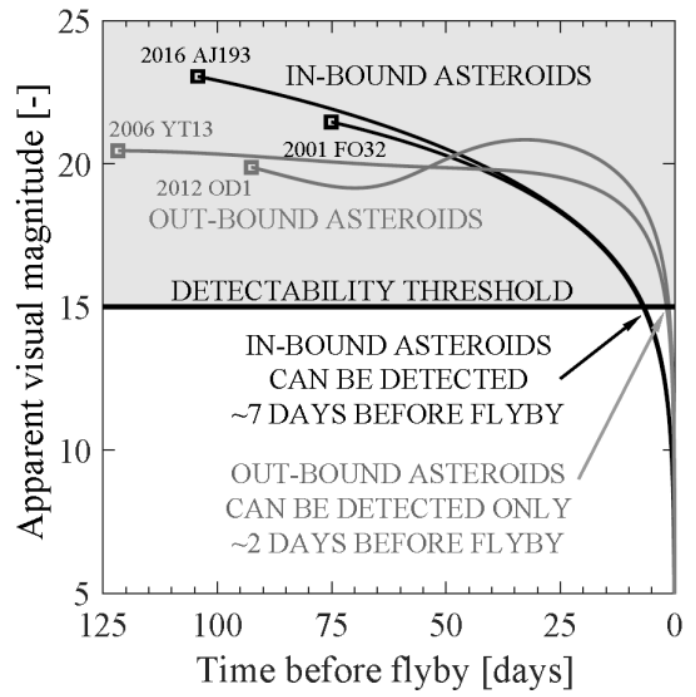

Fig. 7. Apparent magnitude for sample in-bound and outbound asteroids.

performance of almost one order of magnitude with respect to the star tracker included in BCT's XACT unit (i.e., higher update rate and accuracy with compatible form factor). Through a narrow field of view, such CHUs were also considered for the CASTAway asteroid survey mission to achieve high levels of sensitivity in exposure times of a few seconds (Bowles et al., 2017). For redundancy of such an essential component for the mission, an additional $0.125 \mathrm{U}$ star tracker could potentially be allocated within the $0.8 \mathrm{U}$ that was reserved for the science payload, or (if a visual camera is selected as the science payload) a visual imager could be used both as the navigational camera and as the science payload. Such a solution, however, would require the camera to provide both high sensitivity and high resolution images, which may require modification of existing cameras on the market or the development of an alternative solution to meet this new requirement.

It is observed in this analysis that the detectability of asteroids primarily depends on whether the asteroid is moving towards the Sun (in-bound) or away from the Sun (out-bound). In-bound asteroids are more likely to have an illuminated face as observed from the CubeSat, which allows for an earlier detection than for out-bound asteroids. Illustrated in Fig. 7 are the apparent visual magnitudes of four sample asteroids (two in-bound and two out-bound asteroids) as observed from the CubeSat throughout the mission. It is observed that in-bound asteroids can generally be detected $\sim 7$ days before the encounter, whereas out-bound asteroids can be detected only $\sim 2$ days before the encounter. 
Table 4

Reachable asteroids in the 2019-2025 time window (150-day mission span, 80-m/s available $\Delta$ V).

\begin{tabular}{|c|c|c|c|c|c|c|c|c|}
\hline Name & From & $\begin{array}{l}\text { Date of Earth } \\
\text { encounter }\end{array}$ & $\begin{array}{l}\text { Taxonomy } \\
\text { class }^{c}\end{array}$ & $\begin{array}{l}\text { Diameter }^{\mathrm{d}} \\
\text { (m) }\end{array}$ & $\begin{array}{l}\text { Total } \Delta V \\
(\mathbf{m} / \mathbf{s})\end{array}$ & TOF (days) & $\begin{array}{l}\text { Relative } \\
\text { velocity } \\
(\mathbf{k m} / \mathbf{s})\end{array}$ & $\begin{array}{l}\text { Visibility } \\
\text { time } \\
\text { (days) }\end{array}$ \\
\hline 2013 CW32 & \multirow{11}{*}{$\mathrm{L} 2$} & 2019-Jan-29 & & 140 & $64.6 \leftrightarrow 76.0$ & $137.6 \leftrightarrow 150.0$ & 15.8 & 0.4 \\
\hline $2010 \mathrm{CO} 1$ & & 2019-Sep-14 & & 150 & $64.1 \leftrightarrow 68.6$ & $135.7 \leftrightarrow 150.0$ & 13.4 & 0.7 \\
\hline $2000 \mathrm{QW}^{\mathrm{a}}$ & & 2019-Sep-14 & $\mathrm{Q}$ & 380 & 45.5 & 138 & 7.0 & 8.8 \\
\hline $2005 \mathrm{WD}^{\mathrm{a}}$ & & 2019-Nov-11 & & 140 & 73.5 & 136.3 & 16.8 & 0.7 \\
\hline 2015 BK509a & & 2020-Feb-28 & & 110 & 48.8 & 150 & 13.9 & 2.6 \\
\hline 2009 XO & & 2020-May-07 & $\mathrm{X} / \mathrm{Xc}$ & 280 & $17.4 \leftrightarrow 74.7$ & $104.4 \leftrightarrow 150.0$ & 12.2 & 0.1 \\
\hline 2008 TZ3 & & 2020-May-10 & & 290 & $22.5 \leftrightarrow 80.0$ & $107.1 \leftrightarrow 150.0$ & 9.4 & 7.1 \\
\hline 2015 BY310 & & 2021-Mar-10 & Q & 160 & 61.0 & 143.6 & 9.5 & 4.9 \\
\hline 2015 BY310 & & 2023-Mar-07 & $\mathrm{Q}$ & 160 & 53.4 & 150 & 9.7 & 4.9 \\
\hline 1998 HH49 & & 2023-Oct-17 & & 190 & $11.3 \leftrightarrow 64.5$ & $66.7 \leftrightarrow 150.0$ & 14.1 & 0.1 \\
\hline 1998 ST27b & & 2024-Oct-12 & $\mathrm{C}$ & 800,400 & $13.4 \leftrightarrow 69.5$ & $100.0 \leftrightarrow 149.8$ & 17.7 & 5.3 \\
\hline $2010 \mathrm{JG}$ & \multirow{14}{*}{ L1 } & 2019-Nov-12 & & 230 & $45.2 \leftrightarrow 61.7$ & $137.5 \leftrightarrow 144.7$ & 15.9 & 0.7 \\
\hline 2001 FO32 & & 2021-Mar-21 & S & 1000 & $3.4 \leftrightarrow 69.4$ & $76.1 \leftrightarrow 150.0$ & 33.6 & 7 \\
\hline 2008 GO20 & & 2021-Jul-27 & & 120 & $6.1 \leftrightarrow 70.3$ & $73.1 \leftrightarrow 150.0$ & 8.4 & 0.7 \\
\hline 2016 AJ193 & & 2021-Aug-21 & & 630 & $12.3 \leftrightarrow 71.9$ & $105.2 \leftrightarrow 144.0$ & 25.6 & 6.7 \\
\hline 2006 YT13 & & 2022-Jul-19 & $\mathrm{A} / \mathrm{R}$ & 760 & $28.7 \leftrightarrow 73.2$ & $119.5 \leftrightarrow 150.0$ & 23.7 & 1.8 \\
\hline 2016 CZ31 & & 2022-Jul-29 & & 130 & $4.3 \leftrightarrow 57.0$ & $88.9 \leftrightarrow 150.0$ & 15.1 & 2.3 \\
\hline 2014 HK129 & & 2022-Dec-20 & & 210 & $21.8 \leftrightarrow 52.9$ & $126.6 \leftrightarrow 150.0$ & 10.1 & 5.7 \\
\hline 2010 XC15 & & 2022-Dec-27 & & 180 & $2.3 \leftrightarrow 70.9$ & $73.9 \leftrightarrow 150.0$ & 9.5 & 3.5 \\
\hline 2015 DG200 & & 2023-Jan-19 & & 160 & $33.9 \leftrightarrow 70.4$ & $129.7 \leftrightarrow 150$ & 20.5 & 0.1 \\
\hline 2011 AG5 & & 2023-Feb-03 & & 150 & $8.6 \leftrightarrow 66.7$ & $88.2 \leftrightarrow 143.5$ & 9.3 & 5.3 \\
\hline 2012 KY3 & & 2023-Apr-13 & & 730 & $30.3 \leftrightarrow 70.4$ & $121.9 \leftrightarrow 150.0$ & 19.6 & 0.6 \\
\hline 2009 SZ99 $^{a}$ & & 2023-Aug-27 & & 430 & 67.9 & 140.6 & 11.5 & 6 \\
\hline $2011 \mathrm{GA}$ & & 2023-Oct-15 & & 240 & $9.3 \leftrightarrow 61.8$ & $107.6 \leftrightarrow 150.0$ & 17.0 & 0.1 \\
\hline 2012 OD1 & & 2024-Jul-28 & & 660 & $12.2 \leftrightarrow 66.5$ & $105.9 \leftrightarrow 150.0$ & 24.8 & 1.2 \\
\hline
\end{tabular}

${ }^{\text {a}}$ Reachable from only one point along the periodic orbit (out of the nine points that were considered).

${ }^{b}$ Binary asteroid.

${ }^{\mathrm{c}}$ Taxonomy class is specified only when available.

${ }^{\mathrm{d}}$ Estimated diameter derived from absolute magnitude and geometric albedo (assumed value of 0.15 (Morbidelli et al., 2002 )).

The last step in the target selection process finally shows that, out of the 24 asteroids that can be reached, only 10 of them can actually be detected by the on-board star tracker three or more days before the encounter (including asteroid 2015 BY310, which can be encountered in years 2021 and 2023 - see Table 4).
Conclusively, through the analysis described in the current section, a total of 11 asteroid flyby opportunities are identified that could potentially be flown by an autonomous CubeSat between 2019 and 2025 (i.e., nearly two opportunities per year). As previously mentioned, 
further discussion on the importance of detecting the asteroid sufficiently early and its impact on the resulting flyby accuracies can be found in Section 5 .

\section{Navigation strategy and sensitivity analysis}

Once potential asteroid flyby opportunities are identified, it is important to understand whether a CubeSat could actually travel along those flyby trajectories considering the limited performance of its sensors and actuators. The pointing accuracy of its ADCS, for instance, will determine the directional accuracy of the impulsive maneuvers, and errors in the execution of these maneuvers will deviate the CubeSat from its desired path, ultimately resulting in a trajectory that might greatly differ from the nominal, designed trajectory.

The mission is therefore designed to include an additional impulsive maneuver to correct the trajectory of the CubeSat based on its own observations (besides the one or two maneuvers on the designed trajectory). However, and although this final correction maneuver is expected to improve the accuracy of the flyby, the resulting flyby altitudes will still differ from their nominal values. In order to provide additional insight on the accuracy of the flybys that can be achieved by the mission, a sensitivity analysis is performed through Monte Carlo simulations, and it is described in the current section.

As mentioned in Section 2, estimation of the $\mathrm{Cu}$ beSat's position and velocity is performed using only observations of the Sun during cruise and observations of the asteroid before the flyby, and not through radiometric observations from ground stations on the Earth (e.g., NASA's DSN). Observations of the Sun are collected every hour by a coarse sun sensor: the first observation is collected one day after the last design maneuver is performed, and the last observation is collected one day before the asteroid can be detected by the navigation camera for the first time. One hour after the asteroid becomes visible from the CubeSat, observations of the asteroid are then collected instead: one observation every hour until the final correction maneuver is performed.

At the end of the navigation phase, the on-board computer calculates the necessary correction maneuver for the CubeSat to perform a flyby with the same conditions as those on the designed trajectory (same position and same time). This final correction maneuver is performed one hour after the last observation is collected, and it is computed using the differential corrector targeting algorithm described in Section 4. This algorithm outputs the required change in velocity to arrive to the nominal closest approach position (at the nominal time of closest approach) from the estimated position and velocity of the CubeSat. The available $\Delta \mathrm{V}$ for this final maneuver is equal to the remaining $\Delta \mathrm{V}$ after the one or two maneuvers along the designed flyby trajectory.

Although a detailed analysis on the on-board computer capabilities is not performed here, it is worth mentioning that the time allocated between observations, and the one-hour gap between the last observation and the final correction maneuver, is expected to provide the onboard computer with sufficient time to process the observations and to compute the required correction maneuver despite its limited computational power. Time between observations is also allocated for other housekeeping tasks such as performing attitude determination, reorienting the spacecraft, communicating with Earth if necessary, etc.

Estimation of the CubeSat's position and velocity is specifically performed in this analysis through a nonlinear least squares estimator (Wiesel, 2003). During cruise, only directional information between the $\mathrm{Cu}$ beSat and the Sun is available: at each observation time, the coarse sun sensor can provide the estimator with two angles defining the direction vector from the CubeSat to the Sun (according to its own attitude determination knowledge). When observations of the asteroid are collected instead, the navigation camera can provide the estimator with three quantities: the first two elements define the direction from the CubeSat to the asteroid, and the third element provides information on the relative distance between the CubeSat and the asteroid. The brightness of the pixels containing the image of the asteroid, for instance, can provide an estimated value for the apparent visual magnitude of the asteroid (although with limited accuracy). At each observation time then, the third element provided by the navigation camera to the estimator is the apparent visual magnitude of the asteroid as observed from the CubeSat (which provides an implicit measure of the distance between the CubeSat and the asteroid). Alternatively, the relative motion of the asteroid against background stars (i.e., measured parallax) could also provide valuable information to estimate the position and velocity of the CubeSat (Riedel et al., 2000).

It is observed in this analysis that the least squares estimator faces significant difficulties to converge to an appropriate solution using only these measurements. The solution proposed in this work (that is, to improve the convergence properties of the on-board navigation 
algorithm) is to provide the estimator with an a priori estimate of the CubeSat's trajectory and with a corresponding confidence (or covariance) matrix for that estimation. The a priori estimate for the trajectory can simply be the designed trajectory (i.e., nominal, unperturbed trajectory), whereas the covariance matrix associated to that estimation can be generated by a pre-flight, on-ground Monte Carlo simulation: this Monte Carlo simulation should introduce errors in the one or two impulsive maneuvers along the designed trajectory, and in the departure position and velocity from the periodic orbit. Alternatively, more complex navigation campaigns (e.g., more frequent observations, observations of the Earth, Moon, or other planets, etc.) could also improve the accuracy and convergence properties of this estimation process, and consequently result in lower flyby accuracies or allow for accurate autonomous navigation without the need of collecting observations of the target asteroid. A simple navigation strategy, however, is considered in this study to minimize the operational complexity of the mission, and to provide the reader with a simple, yet effective, mission concept.

In order to understand the flyby accuracies that can be achieved by an autonomous CubeSat, Monte Carlo simulations are performed introducing errors in all the impulsive maneuvers (designed maneuvers and final correction maneuver), in the departure position and velocity, and in the observations. Normally distributed errors are introduced in: (1) the direction and magnitude of each impulsive maneuver, according to the thruster and pointing accuracies specified in Table 3 , (2) the initial position and velocity $(3 \sigma$ errors of $10 \mathrm{~km}$ along each component of the position, and $0.1 \mathrm{~m} / \mathrm{s}$ along each component of the velocity), according to the autonomous optical navigation accuracy expected for the LUMIO mission around the Earth-Moon L2 point (Franzese et al., 2018), which could also be complemented by more accurate navigation data from the main spacecraft prior to being deployed, (3) the measured direction vector from the CubeSat to the Sun ( $3 \sigma$ errors of $1 \mathrm{deg})$, according to the modest pointing knowledge that can be provided by the coarse sun sensor integrated in BCT's XACT unit (Caspi, 2017), or by other sun sensors available off-theshelf (Hyperion Technologies B.V., 2017), (4) the measured direction vector from the CubeSat to the asteroid, according to the pointing knowledge specifications in Table 3, and (5) the measured apparent visual magnitude of the asteroid (a conservative $3 \sigma$ error of $30 \%$ ), to account for asteroid modeling errors and navigation camera performance.
The main purpose of this analysis is to demonstrate the capabilities of autonomous CubeSat systems. As such, the sensitivity analysis primarily considers errors due to the limited performance of CubeSat components. Additional sources of error that are not considered in this study include, for instance: (1) uncertainties in the trajectory of the asteroid, and (2) errors induced by the onboard computer using a dynamical model of lower fidelity than the true dynamical model (in a real mission, a full ephemeris model would represent the true dynamical environment, whereas in this study, it is modeled as the CR3BP). Although further analysis is required in this regard, in a real mission (and if sufficient computational power is available) it is recommended for the CubeSat's on-board computer to employ a dynamical model of similar or higher fidelity than the CR3BP (since flyby trajectories may be significantly influenced by the gravitational fields of the Earth and the Moon (Gao, 2013)).

Results from the Monte Carlo simulations are shown in Table 5, which summarizes the flyby accuracies that can be achieved for the identified asteroid flyby opportunities, along with the uncertainty parameter $U$ associated to each asteroid. The $\mathrm{U}$ parameter describes the uncertainty in the orbit of the asteroids: from 0 (very small uncertainty) to 9 (very large uncertainty) (Minor Planet Center, 2012). Unless additional asteroid orbit determination is performed prior to the mission, it is recommended to plan flyby missions to asteroids with low uncertainty parameters.

It has been previously mentioned that the final correction maneuver is implemented at the end of the navigation phase. However, it has not yet been specified when the last observation of the asteroid should be collected, and how this decision affects the resulting flyby accuracies. Two competing factors are involved in this decision process: (1) increasing the extent of the navigation phase improves the estimation of the CubeSat's position and velocity (and consequently the computation of the correction maneuver becomes more accurate), and (2) delaying the correction maneuver increases the required $\Delta \mathrm{V}$ to rectify the trajectory of the CubeSat.

In order to understand how the implementation time of the correction maneuver affects the accuracy of the flybys, Monte Carlo simulations are performed for implementation times every 0.5 days: asteroid $2010 \mathrm{XC} 15$, for instance, is visible for 3.0 days, and 1000 Monte Carlo runs are performed for cases in which the correction maneuver is implemented 0.5 days, 1.0 days, 1.5 days, 2.0 days, 2.5 days, and 3.0 days before the nominal flyby. 


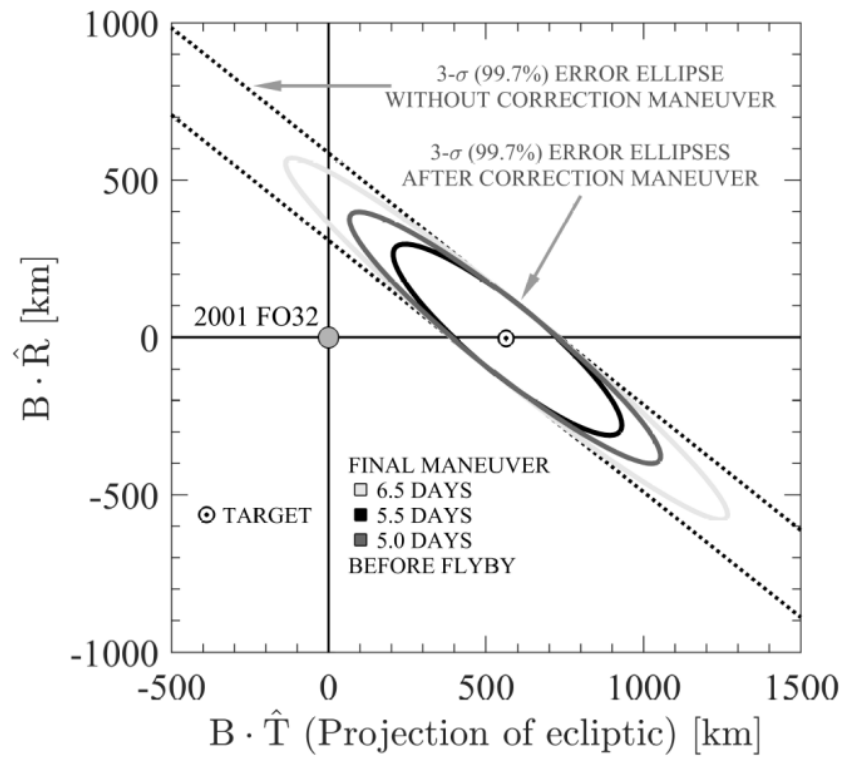

Fig. 8. B-plane error ellipses for asteroid 2001 FO32 and different implementation times of the correction maneuver.

Additionally, these Monte Carlo simulations are performed for all the departure points along the periodic orbit from which the asteroid can be reached with $60 \mathrm{~m} / \mathrm{s}$ of $\Delta \mathrm{V}$ or less (the remaining $>20 \mathrm{~m} / \mathrm{s}$ are reserved for the correction maneuver, and this further constrains the number of accessible asteroids to 9).

As an example, illustrated in Fig. 8 are the resulting Bplane $3 \sigma$ error ellipses for asteroid 2001 FO32 (visible for 7 days - see Table 4). As such, the flyby performed by the CubeSat is expected to be within the enclosed area by these ellipses in $99.7 \%$ of the cases. From this particular departure point, $8.4 \mathrm{~m} / \mathrm{s}$ of $\Delta \mathrm{V}$ are required to reach the asteroid along the designed flyby trajectory, and therefore $71.6 \mathrm{~m} / \mathrm{s}$ remain available for the correction maneuver. The dotted black line illustrates here the error ellipse that would result from a mission with no correction maneuver. This error ellipse expands over $>20,000 \mathrm{~km}( \pm 10,000-\mathrm{km} 3 \sigma$ uncertainty $)$, and is consequently too unreliable for a viable mission (i.e., a final correction maneuver is necessary for the success of the mission). The color-coded solid lines illustrate the resulting error ellipses if the correction maneuver is implemented $6.5,5.5$, and 5.0 days before the flyby. It is observed how the 6.5-day ellipse expands over $1500 \mathrm{~km}$ (since observations of the asteroid where collected for only 0.5 days). The solid black line illustrates the error ellipse with minimum uncertainty: $\pm 350-\mathrm{km} 3 \sigma$ uncertainty, which results from observing the asteroid for 1.5 days (i.e., the correction maneuver is implemented 5.5 days before the flyby). Finally, the error ellipse increases in size $(\sim 1000 \mathrm{~km})$ if the maneuver is performed
Table 5

Achievable flyby accuracies for asteroids visible $>3.0$ days.

\begin{tabular}{|c|c|c|c|}
\hline Name & From & $\begin{array}{l}3 \sigma(99.7 \%) \text { flyby } \\
\text { uncertainty }(\mathbf{k m})^{b}\end{array}$ & $\begin{array}{l}\mathrm{U} \\
\text { parameter }\end{array}$ \\
\hline $2000 \mathrm{QW}^{\mathrm{a}}$ & \multirow{4}{*}{ L2 } & \pm 2484 & 0 \\
\hline $2008 \mathrm{TZ3}$ & & $\pm 337 \leftrightarrow \pm 1771$ & 0 \\
\hline 2015 BY310 & & \pm 12657 & 0 \\
\hline 1998 ST27 & & $\pm 226 \leftrightarrow \pm 851$ & 0 \\
\hline 2001 FO32 & \multirow{5}{*}{ L1 } & $\pm 294 \leftrightarrow \pm 9239$ & 0 \\
\hline 2016 AJ193 & & $\pm 172 \leftrightarrow \pm 770$ & 4 \\
\hline 2014 HK129 & & $\pm 1855 \leftrightarrow \pm 7218$ & 3 \\
\hline 2010 XC15 & & $\pm 137 \leftrightarrow \pm 10407$ & 1 \\
\hline 2011 AG5 & & $\pm 77 \leftrightarrow \pm 702$ & 1 \\
\hline
\end{tabular}

${ }^{a}$ Reachable from only one point along the periodic orbit (out of the nine points that were considered).

${ }^{b}$ Best and worst cases out of all the departure points from which the asteroid can be reached with $>20 \mathrm{~m} / \mathrm{s}$ available for the correction maneuver.

5.0 days before the encounter, since in this case the available $\Delta \mathrm{V}$ for the correction maneuver $(71.6 \mathrm{~m} / \mathrm{s})$ does no longer suffice to fully rectify the trajectory of the CubeSat.

Table 5 summarizes similar results for the 9 asteroids that can be reached, are visible for more than 3 days, and more than $20 \mathrm{~m} / \mathrm{s}$ remain available for the correction maneuver. The results in Table 5 show the range of minimum $3 \sigma$ uncertainty that can be achieved for each asteroid out of all departure points: i.e., the case of minimum uncertainty is selected out of all the maneuver implementation times that were considered; and the cases of smallest and largest uncertainty out of all the departure points are then shown in Table 5.

Moreover, the importance of visibility time becomes particularly evident for asteroids 2000 QW7 and 2015 BY310. A nominal $\Delta \mathrm{V}$ of $45.5 \mathrm{~m} / \mathrm{s}$ is required to encounter asteroid 2000 QW7 in 2019, and $53.4 \mathrm{~m} / \mathrm{s}$ to encounter asteroid 2015 BY310 in 2023 (refer to Table 4). Because only $\sim 30 \mathrm{~m} / \mathrm{s}$ remain available for the correction maneuver, limited flyby accuracies can be achieved for these two asteroids. Asteroid 2015 BY310 is visible for only 4.9 days and poor accuracies can be accomplished: $\pm 12,657 \mathrm{~km} 3 \sigma$ error. Asteroid 2000 QW7 is visible for 8.8 days instead, and a significant improvement in accuracy is observed: $\pm 2484 \mathrm{~km} 3 \sigma$ error. If sufficient $\Delta \mathrm{V}$ and visibility time are available (e.g., asteroid 2011 AG5 with minimum $8.6-\mathrm{m} / \mathrm{s} \Delta \mathrm{V}$ requirement and 
5.3 days of visibility), $3 \sigma$ flyby accuracies as good as $\pm 77 \mathrm{~km}$ are possible.

This work ultimately shows that asteroid flybys comfortably below $1000 \mathrm{~km}$ (or even $500 \mathrm{~km}$ ) can be achieved by an autonomous 3U CubeSat using technology that is currently available. The success of such a mission is, however, contingent to the extent of time that the asteroid is visible from the CubeSat and to the remaining $\Delta \mathrm{V}$ available for the correction maneuver, and it is also dependent on the point of departure from the periodic orbit. Further discussion on the scientific objectives that could be accomplished by such a mission is provided in Section 6.

\section{Potential science return}

Various scientific objectives could be achieved by an asteroid flyby mission depending on the instruments on board the CubeSat. As outlined in Section 3.1, several alternatives for the science payload are available on the market, and although the number of instruments on board ESA's Rosetta spacecraft goes far beyond the payload capacity of the NEARCube mission, its scientific accomplishments (as it orbited comet 67P/Churyumov-Gerasimenko and flew by asteroids Šteins and Lutetia) provide a good example of the possible measurements that a CubeSat could perform.

The Rosetta spacecraft was equipped with narrow and wide angle cameras, ultraviolet, infrared and microwave spectrometers, an ion-neutral mass spectrometer, a fluxgate magnetometer, et cetera (Glassmeier et al., 2007a). Some of the scientific goals covered by Rosetta include characterizing cometary and asteroid exosphere and magnetic field, physical and thermal properties, surface composition and geomorphology, etc.

In the NEARCube mission, UV-VIS-IR spectrometers could allow for the characterization of surface mineralogy, evaluation of space weathering effects, or for taxonomic studies (Reddy et al., 2015). Far-UV and mid-IR spectrometers could enable more complex science objectives such as the search of asteroid exosphere; or (if used for cometary exploration) they could enable the characterization of cometary nucleus and coma composition, nuclear activity, and thermal history (A'Hearn et al., 2010; Coradini et al., 1995). Microwave and mass spectrometers could also allow for the study of cometary outgassing and coma development, characterization of subsurface temperature and structure (Gulkis et al., 2007), analysis of cometary chemical and isotopic composition, and determination of processes for the formation of the atmosphere and ionosphere (Balsiger et al., 2007). A high-resolution magnetometer could also be used for search of asteroid and cometary remnant magnetic fields (Glassmeier et al., 2007b).

As discussed in Section 3.2, approximately $0.8 \mathrm{U}$ is allocated in the NEARCube spacecraft for the science payload. The selection of the science payload might impose additional requirements on the spacecraft besides those highlighted in Table 1, and should be carefully considered during the design of the mission (e.g., a body-mounted magnetometer would require a magnetically clean spacecraft, a high-resolution imager would require a 3-axis stabilized spacecraft during the flyby, particle and field detectors might require a spinning spacecraft, etc.). As an example, the science payload proposed in this study for the NEARCube mission includes SCS Space's 0.6U Gecko imager (SCS Space, 2017), and two Ocean Optics' 0.05U STS spectrometers (Ocean Optics, 2016, 2013b)_UV and VIS, VIS and IR, or UV and IR.

According to the analysis performed in Section 5, flyby altitudes below $500 \mathrm{~km}$ could be achieved using a $3 \mathrm{U}$ CubeSat. Limited spatial resolution could be provided by an on-board camera at such a distance, similar to the spatial resolution that can be accomplished from LEO: $>30 \mathrm{~m}$ at $500 \mathrm{~km}$. Images of the target asteroid will therefore be limited to 10-900 pixels depending on the size of the asteroid (e.g., 100-1000-m asteroids). UV-VIS-IR spectrometers would likely not provide any spatial resolution, but analysis of the incoming light could provide valuable information on the composition of the asteroid, mineral abundances, and space weathering effects. Such observations can also provide an opportunity to support taxonomic studies, and to calibrate and validate ground-based spectral interpretations.

Modest scientific return can therefore be expected from such a mission. The proposed spacecraft design is primarily composed of readily-available, off-the-shelf components, and thus the development and implementation costs of the CubeSat could be comparable to those of a typical LEO CubeSat (i.e., potentially below $\$ 0.5 \mathrm{M}$ (Nervold et al., 2016; SpaceNews, 2017b; Straub, 2012)). Operational costs can be significantly reduced by the autonomous navigation strategy described in this study, and ground segment operations can consequently be limited to seldom transmission of telemetry and commands (if spacecraft operations are not fully automated), and to the downlink of scientific data generated during the flyby. A number of ground stations and commercial companies are currently developing affordable solutions 
for deep-space communications as an alternative to governmental networks like DSN and ESTRACK (ATLAS Space Operations, 2017; ESA, 2018). Potentially, the downlink of science data may be possible at even less than $\$ 500$ per pass (SpaceNews, 2017a), although this will highly depend on the required antenna size. For comparison, the overall cost of the Rosetta mission was $\$ 1.5$ billion (ESA, 2014), and the cost of the $563-\mathrm{kg}$ NEAR Shoemaker mission was \$220 million, which landed on asteroid 433 Eros after almost a year of onorbit operations (\$113-million development costs and \$60-million operations costs), and whose science payload included a multispectral high-resolution camera, a magnetometer, near-IR and X-ray/Gamma-ray spectrometers, and a laser altimeter (NASA, 2005).

Advances in CubeSat technology, using a larger spacecraft (e.g., 6U CubeSat), or a more detailed analysis of the $3 \mathrm{U}$ mission concept could further improve the quality of the mission and its scientific return, potentially enabling lower flyby altitudes, lower relative velocities, or even orbiting or landing on asteroids. A larger CubeSat platform, for instance, could allow for larger $\Delta \mathrm{V}$ capabilities to enable access to more asteroids or to further correct the trajectory of the CubeSat before the flyby, it could allow for higher redundancy in the system, improve the capabilities of critical components for the mission (e.g., star tracker, on-board computer, generated power, etc.), or it could allocate additional sensors for autonomous navigation (e.g., fine sun sensor, a redundant star tracker, a high-sensitivity narrow angle camera, etc.) or a larger and more capable science payload (e.g., higher-resolution visual camera, mass spectrometer, etc.). A $6 \mathrm{U}$ mission could also provide higher flexibility to the mission and relax some of the constraints imposed by the $3 \mathrm{U}$ platform. For example, more accurate navigation could be performed (which could allow for lower flyby altitudes), accurate navigation might be possible without observations of the target asteroid (i.e., flyby missions would not be constrained to asteroids that are visible prior to the flyby), or knowledge on the trajectory of the asteroid could be refined prior to the flyby (i.e., flyby missions to asteroids with larger uncertainties in their trajectory might become viable).

Through a simple mission concept and a preliminary analysis, this work demonstrates that CubeSats can today provide a real low-cost solution for small-body planetary exploration, and reduce not only the development and implementation costs as compared to traditional missions, but also operations costs through autonomous navigation techniques. And although only mod- est scientific return can be expected from such a mission, CubeSats can cost-effectively and significantly support larger scientific missions, or efforts in the asteroid mining industry (e.g., they could be used to characterize potential target asteroids, to collect complementary measurements, etc.).

\section{Concluding remarks}

In order to demonstrate the capability of current $\mathrm{Cu}$ beSat technology to provide a real low-cost solution for small-body planetary exploration, a 3U CubeSat mission is designed to flyby near-Earth asteroids using autonomous navigation. Asteroid flyby opportunities are identified that could be flown by an autonomous $3 \mathrm{U} \mathrm{Cu}-$ beSat between years 2019 and 2025, using only observations of the Sun during cruise and observations of the target asteroid prior to the flyby (instead of relying on ground stations for navigation support). Such a mission leverages piggyback opportunities from a larger spacecraft orbiting the first or the second Sun-Earth Lagrange points, and flyby altitudes even below $500 \mathrm{~km}$ are found possible based on the current state of CubeSat components.

By means of lower development, implementation, and ground operations costs, the present work shows that interplanetary CubeSats can be used to explore near-Earth asteroids at a significantly lower cost than traditional missions. The scientific return of such missions is primarily limited to, for instance, medium resolution images and spectroscopic observations of the asteroid, but it could be further extended by upcoming advancements in CubeSat technology, by the use of a larger CubeSat platform, or through a more detailed analysis of the $3 \mathrm{U}$ mission concept (e.g., implementing a more complex and accurate navigation campaign). Similar results are also anticipated for other piggyback opportunities (e.g., for a deployment in an Earth-Moon transfer trajectory), although further analysis is required to confidently extend these remarks.

Future efforts will as well focus on the use of higherfidelity dynamical models (i.e., ephemeris model) for the trajectory design analysis, on the evaluation of more sophisticated autonomous navigation strategies and models (e.g., use observations of the Earth-Moon system and other planetary bodies if visible, which could improve the accuracy of the on-board estimation and thus the accuracy of the flybys), on the use of more realistic sensor-actuator models (e.g., abandon the impulsive maneuver assumption), on a more detailed analysis of the communications and power systems (i.e., link 
budget analysis, power budget), and on the analysis of a $6 \mathrm{U}$ CubeSat mission instead.

Ultimately, this study shows that autonomous $3 \mathrm{U} \mathrm{Cu}-$ beSats do provide a low-cost alternative to the planetary science community, and can be considered for future missions to further our knowledge on the origins of the Solar System, or to support the efforts of the asteroid mining enterprise.

\section{References}

A'Hearn, M.F., Feaga, L.M., Bertaux, J.L., Feldman, P.D., Parker, J.W., Slater, D.C., Steffl, A.J., Alan Stern, S., Throop, H., Versteeg, M., Weaver, H.A., Uwe Keller, H., 2010. The Far-Ultraviolet Albedo of Šteins Measured with Rosetta-ALICE. Planetary and Space Science 58, 1088-1096. https://doi.org/10.1016/j.pss.2010.03.005

Arnold, S.S., Nuzzaci, R., Gordon-Ross, A., 2012. Energy budgeting for CubeSats with an integrated FPGA, in: IEEE Aerospace Conference. Institute of Electrical and Electronics Engineers Inc., Big Sky, MT, USA. https://doi.org/10.1109/AERO.2012.6187240

Aslan, A.R., Yağc1, H.B., Umit, M.E., Sofyalı, A., Bas, M.E., Uludag, M.S., Ozen, O.E., Aksulu, M.D., Yakut, E., Oran, C., Suer, M., Akyol, İ.A., Ecevit, A.B., Ersöz, M.S., Öz, İ., Gülgönül, Ş., Dinç, B., Dengiz, T., 2013. Development of a LEO Communication CubeSat, in: 2013 6th International Conference on Recent Advances in Space Technologies (RAST). pp. 637-641. https://doi.org/10.1109/RAST.2013.6581288

ATLAS Space Operations, 2017. ATLAS Introduces New Commercial Deep Space Network \& Pathfinder Mission [WWW Document]. URL http://www.atlasground.com/posts/atlasintroduces-new-commercial-deep-space-network/ (accessed 6.28.18).

Balsiger, H., Altwegg, K., Bochsler, P., Eberhardt, P., Fischer, J., Graf, S., Jäckel, A., Kopp, E., Langer, U., Mildner, M., Müller, J., Riesen, T., Rubin, M., Scherer, S., Wurz, P., Wüthrich, S., Arijs, E., Delanoye, S., De Keyser, J., Neefs, E., Nevejans, D., Rème, H., Aoustin, C., Mazelle, C., Médale, J.L., Sauvaud, J.A., Berthelier, J.J., Bertaux, J.L., Duvet, L., Illiano, J.M., Fuselier, S.A., Ghielmetti, A.G., Magoncelli, T., Shelley, E.G., Korth, A., Heerlein, K., Lauche, H., Livi, S., Loose, A., Mall, U., Wilken, B., Gliem, F., Fiethe, B., Gombosi,
T.I., Block, B., Carignan, G.R., Fisk, L.A., Waite, J.H., Young, D.T., Wollnik, H., 2007. Rosina Rosetta Orbiter Spectrometer for Ion and Neutral Analysis. Space Science Reviews 128, 745-801. https://doi.org/10.1007/s11214-006-8335-3

Barden, B., Howell, K., Lo, M., 1996. Application of Dynamical Systems Theory to Trajectory Design for a Libration Point Mission, in: Astrodynamics Conference, Guidance, Navigation, and Control and Co-Located Conferences. American Institute of Aeronautics and Astronautics. https://doi.org/doi:10.2514/6.1996-3602

Barth, J.L., Isaacs, J.C., Poivey, C., 2000. The Radiation Environment for the Next Generation Space Telescope. Greenbelt, MD, USA.

Bekker, D.L., Werne, T.A., Wilson, T.O., Pingree, P.J., Dontchev, K., Heywood, M., Ramos, R., Freyberg, B., Saca, F., Gilchrist, B., Gallimore, A., Cutler, J., 2010. A CubeSat Design to Validate the Virtex-5 FPGA for Spaceborne Image Processing, in: IEEE Aerospace. Institute of Electrical and Electronics Engineers Inc., Big Sky, MT, USA, pp. 1-9. https://doi.org/10.1109/AERO.2010.5446700

Blackwell, W.J., 2015. The MicroMAS and MiRaTA CubeSat Atmospheric Profiling Missions, in: International Microwave Symposium. Institute of Electrical and Electronics Engineers Inc., Lexington, MA, USA. https://doi.org/10.1109/MWSYM.2015.7166742

Blue Canyon Technologies, 2017. Attitude Determination \& Control Systems. Boulder, CO, USA.

Bonin, G., Foulds, C., Armitage, S., Faber, D., 2016. Prospector-1: The First Commercial Small Spacecraft Mission to an Asteroid, in: 30th Annual AIAA/USU Conference on Small Satellites. Logan, UT, USA, pp. 1-11.

Bourdarie, S., Xapsos, M., 2008. The Near-Earth Space Radiation Environment. IEEE Transactions on Nuclear Science 55, 1810-1832. https://doi.org/10.1109/TNS.2008.2001409

Bowell, E., Hapke, B., Lumme, K., Harris, A.W., Domingue, D., Peltoniemi, J., 1988. Application of Photometric Models to Asteroids. Asteroids II 524-556.

Bowles, N.E., Snodgrass, C., Gibbings, A., Sanchez, J.P., Arnold, J.A., Eccleston, P., Andert, T., Probst, A., Naletto, G., Vandaele, A.C., de Leon, J., 
Nathues, A., Thomas, I.R., Thomas, N., Jorda, L., Da Deppo, V., Haack, H., Green, S.F., Carry, B., Donaldson Hanna, K.L., Leif Jorgensen, J., Kereszturi, A., DeMeo, F.E., Patel, M.R., Davies, J.K., Clarke, F., Kinch, K., Guilbert-Lepoutre, A., Agarwal, J., Rivkin, A.S., Pravec, P., Fornasier, S., Granvik, M., Jones, R.H., Murdoch, N., Joy, K.H., Pascale, E., Tecza, M., Barnes, J.M., Licandro, J., Greenhagen, B.T., Calcutt, S.B., Marriner, C.M., Warren, T., Tosh, I., 2017. CASTAway: An Asteroid Main Belt Tour and Survey. Advances in Space Research. https://doi.org/10.1016/j.asr.2017.10.021

Bunce, D.T., Bassett, K.P., Ghosh, A.R.M., Barnett, P.R., Haken, D.M., Vrkljan, S., Jagannatha, R., Silva, T., Coverstone, V.L., 2016. Microvascular Composite Radiators for Small Spacecraft Thermal Management Systems, in: 30th Annual AIAA/USU Conference on Small Satellites. Logan, UT, USA.

Buonaiuto, N., Kief, C., Louie, M., Aarestad, J., Zufelt, B., Mital, R., Mateik, D., Sivilli, R., Bhopale, A., 2017. Satellite Identification Imaging for Small Satellites Using NVIDIA, in: Small Satellite Conference. Logan, UT, USA.

Cahoy, K., Allan, G., Lee, Z., Hein, A., Kennedy, A., Lee, M., Main, E., Blackwell, B., 2017. Integration and Test of the Microwave Radiometer Technology Acceleration (MiRaTA) CubeSat, in: 31st Annual AIAA/USU Conference on Small Satellites. Logan, UT, USA.

Calla, P., Fries, D., Welch, C., Downer, B., 2017. Lowcost Asteroid Mining Using Small Spacecraft, IAC 2017 Adelaide, Australia.

Caspi, A., 2017. State of the Art: MinXSS CubeSat Performance and CubIXSS Future Needs, in: Keck Institute for Space Studies Optical Communication on SmallSats Optical Communication on SmallSats. Pasadena, CA, USA.

Castillo-Rogez, J., Johnson, L., Abell, P., Bleacher, J., Boland, J., Farrell, B., Graham, L., Thompson, D., 2014. Near Earth Asteroid Scout Mission, in: 11th Meeting of the NASA Small Bodies Assessment Group. Washington, D.C., USA, USA.

Celotti, L., Solyga, M., Nadalini, R., Kravets, V., Khairnasov, S., Baturkin, V., Lange, C., Findlay, R., Ziach, C., Ho, T., 2015. MASCOT Thermal Subsystem Design Challenges and Solution for Contrasting Requirements, in: 45th International
Conference on Environmental Systems. Bellevue, WA, USA.

Chaudery, R.A., 2014. QB50 INMS Science Unit Interface Control Document. London, UK.

Cheung, K.-M., Lee, C., Waldherr, S., Lanucara, M., Malphrus, B.K., Dove, W., 2016. Architecture and Concept of Operation of Next-Generation Ground Network for Communications and Tracking of Interplanetary Smallsats, in: AIAA SpaceOps Conference. Daejeon, South Korea, pp. 1-32. https://doi.org/10.2514/6.2016-2401

Cheung, K.-M., Lee, C., Waldherr, S., Lanucara, M., Malphrus, B.K., Dove, W., 2015. Next-Generation Ground Network for Communications and Tracking of Interplanetary Smallsats, The Interplanetary Network Progress Report. https://doi.org/10.2514/6.2016-2401

Clyde Space, 2017. CPUT X-band Transmitter [WWW Document]. URL https://www.clyde.space/products/66-cput-xbandtransmitter (accessed 1.16.18).

Clyde Space, 2016a. 3U Single-Deployable Solar Array (Short Edge) [WWW Document]. URL https://www.clyde.space/products/28-3usingledeployable-solar-array-short-edge (accessed 1.16.18).

Clyde Space, 2016b. Clyde Space 3U Power Bundle A: EPS + 30Whr Battery [WWW Document]. URL https://www.clyde.space/products/39-cs-3u-

power-bundle-a-eps-30whr-battery (accessed 2.2.18).

Coradini, A., Capaccioni, F., Capria, M.T., Cerroni, P., De Sanctis, M.C., Magni, G., Bonsignori, R., Reininger, F., Encrenaz, T., Drossart, P., Semery, A., Arnold, G., Michaelis, H., Taylor, F.W., Calcutt, S.B., Vellacott, T.J., Venters, P., Watkins, R.E.J., 1995. VIRTIS, Visible Infrared Thermal Imaging Spectrometer for the ROSETTA Mission, in: 1995 International Geoscience and Remote Sensing Symposium, IGARSS '95. Quantitative Remote Sensing for Science and Applications. pp. 1604-1606.

https://doi.org/10.1109/IGARSS.1995.521822

Crystalspace, 2015. Crystalspace CAM1U CubeSat Camera [WWW Document]. URL http://crystalspace.eu/products/crystalspace-clucubesat-camera/ (accessed 1.16.18).

CU Aerospace, 2017. Propulsion Unit for CubeSats. 
Champaign, IL, USA.

CU Aerospace, 2014. Propulsion Unit for CubeSats. Champaign, IL, USA.

Cubic Aerospace, 2017. Massively Multi-Core Payload Processor. Reston, VA, USA.

Deb, K., Pratap, A., Agarwal, S., Meyarivan, T., 2002. A Fast and Elitist Multiobjective Genetic Algorithm: NSGA-II. IEEE Transactions on Evolutionary Computation 6, 182-197. https://doi.org/10.1109/4235.996017

Deep Space Industries, 2016. Deep Space Industries Comet- $1^{\mathrm{TM}}$ CubeSat and Microsatellite Water Thrusters. Mountain View, CA, USA.

DHV Technology, 2015. Solar panels for aerospace applications. Málaga, Spain.

Domingo, V., Fleck, B., Poland, A.I., 1995. The SOHO Mission: An Overview. Solar Physics. https://doi.org/10.1007/BF00733425

Duncan, C.B., Smith, A.E., Aguirre, F.H., 2014. Iris Transponder - Communications and Navigation for Deep Space, in: 28th Annual AIAA/USU Conference on Small Satellites.

Dymock, R., 2007. The H and G Magnitude System for Asteroids. Journal of the British Astronomical Association $342-343$. https://doi.org/10.1007/s13398-014-0173-7.2

EADS Astrium Ltd, 2005. LISA Pathfinder: General Design and Interface Requirements Specification (GDIR). Stevenage, UK.

EnduroSat, 2016. EnduroSat CubeSat 3U Solar Panel $\mathrm{X} / \mathrm{Y} \quad$ [WWW Document]. URL https://www.endurosat.com/products/cubesat-3usolar-panel-x-y/ (accessed 1.16.18).

ESA, 2018. Goonhilly Goes Deep Space [WWW Document]. URL https://m.esa.int/Our_Activities/Operations/Estrac k/Goonhilly_goes_deep_space (accessed 6.28.18).

ESA, 2017a. PLATO - Definition Study Report.

ESA, 2017b. ARIEL - Assessment Study Report.

ESA, 2014. Rosetta Press Kit - Landing on a Comet.

Escobar, E., Diaz, M., Zagal, J.C., 2016. Evolutionary Design of a Satellite Thermal Control System: Real Experiments for a CubeSat Mission. Applied Thermal Engineering 105, 490-500. https://doi.org/10.1016/j.applthermaleng.2016.03.
024

EXA, 2016. EXA BA0X: 1U High Capacity Battery Arrays. Guayaquil, Ecuador.

Farrah, D., Smith, K.E., Ardila, D., Bradford, C.M., Dipirro, M., Ferkinhoff, C., Glenn, J., Goldsmith, P., Leisawitz, D., Nikola, T., Rangwala, N., Rinehart, S.A., Staguhn, J., Zemcov, M., Zmuidzinas, J., Bartlett, J., Carey, S., Fischer, W.J., Kamenetzky, J., Kartaltepe, J., Lacy, M., Lis, D.C., Lopez-Rodriguez, E., MacGregor, M., Moseley, S.H., Murphy, E.J., Rhodes, A., Richter, M., Rigopoulou, D., Sanders, D., Sankrit, R., Savini, G., Smith, J.-D., Stierwalt, S., 2017. Review: Far-Infrared Instrumentation and Technology Development for the Next Decade. Journal of Astronomical Telescopes, Instruments, and Systems.

Fernandez, M.A., Guillois, G., Richard, Y., Issler, J.-L., Lafabrie, P., Gaboriaud, A., Evans, D., Walker, R., Koudelka, O., Romano, P., Hansen, K.T., Gerhardt, D., 2015. A Game-changing Radio Communication Architecture for Cube/Nano Satellites, in: 29th Annual AIAA/USU Conference on Small Satellites. pp. 1-10.

Franzese, V., Di Lizia, P., Topputo, F., 2018. Autonomous Optical Navigation for LUMIO Mission. 2018 Space Flight Mechanics Meeting. https://doi.org/10.2514/6.2018-1977

Gao, Y., 2013. Near-Earth Asteroid Flyby Trajectories from the Sun-Earth L2 for Chang'e-2's Extended Flight. Acta Mechanica Sinica 29, 123-131. https://doi.org/10.1007/s10409-013-0011-8

German Aerospace Center, 2016. DLR Institute of Space Systems, Status Report 2007-2016, Part I. Bremen, Germany.

Gilmore, D.G., 2002. Spacecraft Thermal Control Handbook Volume I: Fundamental Technologies.

Glassmeier, K.H., Boehnhardt, H., Koschny, D., Kührt, E., Richter, I., 2007a. The Rosetta Mission: Flying Towards the Origin of the Solar System. Space Science Reviews 128, 1-21. https://doi.org/10.1007/s11214-006-9140-8

Glassmeier, K.H., Richter, I., Diedrich, A., Musmann, G., Auster, U., Motschmann, U., Balogh, A., Carr, C., Cupido, E., Coates, A., Rother, M., Schwingenschuh, K., Szegö, K., Tsurutani, B., 2007b. RPC-MAG the Fluxgate Magnetometer in the ROSETTA Plasma Consortium. Space Science 
Reviews 128, 649-670. https://doi.org/10.1007/s11214-006-9114-X

Goel, A., Krishnamoorthy, S., Swenson, T., West, S., Li, A., Crew, A., Phillips, D.J., Screve, A., Close, S., 2017. Design for CubeSat-based Dust and Radiation Studies at Europa. Acta Astronautica 136, 204-218. https://doi.org/10.1016/j.actaastro.2017.03.016

Gómez, G., Jorba, A., Masdemont, J., Simó, C., 1993. Study of the Transfer from the Earth to a Halo Orbit around the Equilibrium Point L1. Celestial Mechanics \& Dynamical Astronomy 56, 541-562. https://doi.org/10.1007/BF00696185

Gómez, G., Masdemont, J., 2000. Some Zero Cost Transfers between Libration Point Orbits. Advances in the Astronautical Sciences 105 II, 1199-1216.

GomSpace, 2017. C1U NanoCam Datasheet. Aalborg, Denmark.

GomSpace, 2014. GomSpace NanoPower P-series Datasheet.

Goudas, C.L., 1963. Three-dimensional Periodic Orbits and their Stability. Icarus 2, 1-18.

Greenhouse, M.A., Mather, J.C., Clampin, M., Doyon, R., Flanagan, K.A., Franx, M., Hammel, H.B., Hutchings, J.B., Jakobsen, P., Lilly, S.J., Lunine, J.I., McCaughrean, M.J., Mountain, M., Rieke, G.H., Rieke, M.J., Sonneborn, G., Stiavelli, M., Windhorst, R., Wright, G.S., 2011. The James Webb Space Telescope: Mission Overview and Status, in: 2nd International Conference on Space Technology, ICST 2011. https://doi.org/10.1109/ICSpT.2011.6064655

Gulkis, S., Frerking, M., Crovisier, J., Beaudin, G., Hartogh, P., Encrenaz, P., Koch, T., Kahn, C., Salinas, Y., Nowicki, R., Irigoyen, R., Janssen, M., Stek, P., Hofstadter, M., Allen, M., Backus, C., Kamp, L., Jarchow, C., Steinmetz, E., Deschamps, A., Krieg, J., Gheudin, M., Bockelée-Morvan, D., Biver, N., Encrenaz, T., Despois, D., Ip, W., Lellouch, E., Mann, I., Muhleman, D., Rauer, H., Schloerb, P., Spilker, T., 2007. MIRO: Microwave Instrument for Rosetta Orbiter. Space Science Reviews 128, 561-597. https://doi.org/10.1007/s11214-006-9032-y

Guo, J., Monas, L., Gill, E., 2014. Statistical Analysis and Modelling of Small Satellite Reliability. Acta Astronautica. https://doi.org/10.1016/j.actaastro.2014.01.018

Hannu Leppinen, Antti Kestilä, Pauli Pihajoki, Jukka Jokelainen, T.H., 2014. On-board Data Handling for Ambitious Nanosatellite Missions Using Automotive-grade Lockstep Microcontrollers, in: Small Satellites, Systems and Services Symposium. Mallorca, Spain, pp. 1-10.

Hegel, D., 2016. FlexBus - A 6U Cubesat Platform for Any Mission, in: 13th Annual CubeSat Developers Workshop. San Luis Obispo, CA, USA.

Heidt, H., Puig-Suari, J., Moore, A.S., Nakasuka, S., Twiggs, R.J., 2000. CubeSat: A New Generation of Picosatellite for Education and Industry LowCost Space Experimentation, in: 14th Annual AIAA/USU Conference on Small Satellites. Logan, UT, USA, pp. 1-19.

Hodges, R.E., Chahat, N., Hoppe, D.J., Vacchione, J.D., 2017. A Deployable High-Gain Antenna Bound for Mars: Developing a New Folded-panel Reflectarray for the First CubeSat Mission to Mars. IEEE Antennas and Propagation Magazine 59 , $39-49$ https://doi.org/10.1109/MAP.2017.2655561

Howell, K., 1984. Three-Dimensional, Periodic, "Halo" Orbits. Celestial Mechanics 32, 53. https://doi.org/10.1007/BF01358403

Howell, K.C., 2001. Families of Orbits in the Vicinity of the Collinear Libration Points. Journal of the Astronautical Sciences 49, 107-125.

Howell, K.C., Mains, D.L., Barden, B.T., 1994. Transfer Trajectories from Earth Parking Orbits to SunEarth Halo Orbits, in: Advances in the Astronautical Sciences. pp. 399-410.

Howell, K.C., Pernicka, H.J., 1987. Numerical Determination of Lissajous Trajectories in the Restricted Three-body Problem. Celestial Mechanics 41, 107-124. https://doi.org/10.1007/BF01238756

Hunter, R., 2015. CubeSats and Science Return, in: The Hosted Payload and SmallSat Summit. Washington, D.C., USA, pp. 1-37.

Hyperion Technologies B.V., 2017. Hyperion Technologies SS200 Sun Sensor. Delft, Netherlands.

Hyperion Technologies B.V., 2016a. Hyperion Technologies iADCS100 Attitude Determination and Control System. Delft, Netherlands. 
Hyperion Technologies B.V., 2016b. Hyperion Technologies ST200 Star Tracker. Delft, Netherlands.

Innovative Solutions in Space, 2013. ISIS Examples of Umbillical Connectors. Delft, Netherlands.

Johnson, M., Bonalsky1, T., Cameron, T., Chornay, D., Clagett, C., Cudmore, A., Ericsson, A., Hesh, S., Jones, S., Kepko, L., Paschalidis, N., Rodriguez, J., Sittler, E., Starin, S., Santos, L., Sheikh, S., Uribe, P., Zesta, E., 2015. Dellingr - A Path to Compelling Science with CubeSats.

Jørgensen, P.S., Jørgensen, J.L., Denver, T., van den Braembuche, P., 2005. The Micro Advanced Stellar Compass for ESA's PROBA 2 Mission. Proceedings of the 5th International Symposium of the IAA on Small Satellites for Earth Observation 299-303.

Jove-Casulleras, R., Camps, A., Ramos, J., 2012. Cubesat-based Demonstrator for Optical Earth Observation, in: 2012 IEEE International Geoscience and Remote Sensing Symposium. pp. 1505-1508.

https://doi.org/10.1109/IGARSS.2012.6351249

Kahn, P., Imken, T., Elliott, J., Sherwood, B., Frick, A., Sheldon, D., Lunine, J., 2017. Environmental Design Implications for Two Deep Space SmallSats. Acta Astronautica 139, 390-395. https://doi.org/10.1016/j.actaastro.2017.07.025

Kang, S.J., Oh, H.U., 2016. On-Orbit Thermal Design and Validation of $1 \mathrm{U}$ Standardized CubeSat of STEP Cube Lab. International Journal of Aerospace Engineering 2016. https://doi.org/10.1155/2016/4213189

Katke, T., Rathod, A., Marne, A., Prabhune, B., Kothawala, A., Wachche, S., 2016. Thermal System Design and Orbital Analysis of Swayam: An Overview of the Design Principles and Technical Considerations, in: 67th International Astronautical Congress, IAC 2016. International Astronautical Federation, IAF, Pune, India.

Klesh, A., Krajewski, J., 2015. MarCO: CubeSats to Mars in 2016, in: 29th Annual AIAA/USU Small Satellite Conference. pp. 1-7.

Klesh, A.T., Baker, J., Castillo-Rogez, J., Halatek, L., Murphy, N., Raymond, C., Bellardo, J., Cutler, J., Lightsey, G., 2013a. INSPIRE: Interplanetary NanoSpacecraft Pathfinder In Relevant Environment, in: 27th Annual AIAA/USU
Conference on Small Satellites. Logan, UT, USA.

Klesh, A.T., Baker, J.D., Bellardo, J., Castillo-Rogez, J., Cutler, J., Halatek, L., Lightsey, E.G., Murphy, N., Raymond, C., 2013b. INSPIRE: Interplanetary NanoSpacecraft Pathfinder in Relevant Environment, in: AIAA SPACE Conference and Exposition. San Diego, CA, USA, pp. 1-6. https://doi.org/10.2514/6.2013-5323

Klofas, B., 2006. Amateur Radio and the CubeSat Community, in: AMSAT-NA Space Symposium. San Francisco, CA, USA.

Klofas, B., Leveque, K., 2012. The Future of CubeSat Communications: Transitioning Away from Amateur Radio Frequencies for High-speed Downlinks, in: AMSAT Space Symposium.

Koon, W.S., Lo, M.W., Marsden, J.E., Ross, S.D., 2011. Dynamical Systems, the Three-Body Problem and Space Mission Design. Marsden Books, Pasadena, CA, USA. https://doi.org/doi:10.1142/9789812792617_0222

Lagerkvist, C.-I., Magnusson, P., 1990. Analysis of Asteroid Lightcurves. II. Phase Curves in a Generalized HG-System. Astronomy and Astrophysics Supplement Series 86, 119-165.

Langer, M., Bouwmeester, J., 2016. Reliability of CubeSats - Statistical Data, Developers' Beliefs and the Way Forward, in: 30th Annual AIAA/USU Conference on Small Satellites. p. SSC16-X-2.

Laureijs, R., Racca, G., Stagnaro, L., Salvignol, J.-C., Lorenzo Alvarez, J., Saavedra Criado, G., Gaspar Venancio, L., Short, A., Strada, P., Colombo, C., Buenadicha, G., Hoar, J., Kohley, R., Vavrek, R., Mellier, Y., Berthe, M., Amiaux, J., Cropper, M., Niemi, S., Pottinger, S., Ealet, A., Jahnke, K., Maciaszek, T., Pasian, F., Sauvage, M., Wachter, S., Israelsson, U., Holmes, W., Seiffert, M., Cazaubiel, V., Anselmi, A., Musi, P., 2014. Euclid Mission Status, in: Proceedings of SPIE - The International Society for Optical Engineering. https://doi.org/10.1117/12.2054883

Lemmer, K., 2017. Propulsion for CubeSats. Acta Astronautica 134, 231-243. https://doi.org/10.1016/j.actaastro.2017.01.048

Lokman, A.H., Soh, P.J., Azemi, S.N., Lago, H., Podilchak, S.K., Chalermwisutkul, S., Jamlos, M.F., Al-Hadi, A.A., Akkaraekthalin, P., Gao, S., 2017. A Review of Antennas for Picosatellite Applications. International Journal of Antennas 
and https://doi.org/10.1155/2017/4940656

Lorenz, C.G., Case, A.W., Pekosh, J.D., Ahern, D.M., Bernhardt, T., Butt, S., Coverstone, V.L., Eiler, E.A., Ghosh, A., Haddox, P., Kite, A., Lohan, K., Santana, L.F., Santos, D., 2015. An Atmosphere and Plume Explorer of the Jovian System, in: AIAA SPACE 2015 Conference and Exposition. Pasadena, CA, USA, pp. 1-10. https://doi.org/10.2514/6.2015-4535

Marchal, C., 1990. The Three-body Problem, Studies in Astronautics. Elsevier.

Marinan, A., Castillo-Rogez, J., Johnson, L., Dervan, J., Seybold, C., Betts, E., 2017. Near Earth Asteroid (NEA) Scout CubeSat Mission, in: 12th Low-Cost Planetary Missions Conference. Pasadena, CA.

Maryland Aerospace, 2016. MAI-400 1/2U CubeSat ADACS. Crofton, MD, USA.

Mason, J.P., Baumgart, M., Rogler, B., Downs, C., Williams, M., Woods, T.N., Palo, S., Chamberlin, P.C., Solomon, S., Jones, A., Li, X., Kohnert, R., Caspi, A., 2017. MinXSS-1 CubeSat On-Orbit Pointing and Power Performance: The First Flight of the Blue Canyon Technologies XACT 3-axis Attitude Determination and Control System. Journal of Small Satellites.

Maurer, R.H., Fraeman, M.E., Martin, M.N., Roth, D.R., 2008. Harsh Environments: Space Radiation Environment, Effects, and Mitigation. Johns Hopkins APL Technical Digest 28, 17-29.

McNamara, P., Racca, G., 2009. Introduction To LISA Pathfinder.

Mcnutt, L., Johnson, L., Clardy, D., 2014. Near-Earth Asteroid Scout, in: AIAA SPACE 2014 Conference and Exposition. pp. 1-9. https://doi.org/10.2514/6.2014-4435

Mehrparvar, A., 2014. Cubesat Design Specification (CDS), The CubeSat Program, California Polytechnic State https://doi.org/http://dx.doi.org/10.1155/2015/681 901

Michel, P., Cheng, A., Küppers, M., Pravec, P., Blum, J., Delbo, M., Green, S.F., Rosenblatt, P., Tsiganis, K., Vincent, J.B., Biele, J., Ciarletti, V., Hérique, A., Ulamec, S., Carnelli, I., Galvez, A., Benner, L., Naidu, S.P., Barnouin, O.S., Richardson, D.C., Rivkin, A., Scheirich, P., Moskovitz, N., Thirouin,
A., Schwartz, S.R., Campo Bagatin, A., Yu, Y., 2016. Science Case for the Asteroid Impact Mission (AIM): A Component of the Asteroid Impact \& Deflection Assessment (AIDA) Mission. Advances in Space Research 57, 2529-2547. https://doi.org/10.1016/j.asr.2016.03.031

Miles, D.M., Mann, I.R., Ciurzynski, M., Barona, D., Narod, B.B., Bennest, J.R., Pakhotin, I.P., Kale, A., Bruner, B., Nokes, C.D.A., Cupido, C., Haluza-DeLay, T., Elliott, D.G., Milling, D.K., 2016. A Miniature, Low-Power Scientific Fluxgate Magnetometer: A Stepping-Stone to Cube-Satellite Constellation Missions. Journal of Geophysical Research: Space Physics 121, 11,839-11,860.

https://doi.org/10.1002/2016JA023147

Minor Planet Center, 2012. Uncertainty Parameter U and Orbit Quality Codes [WWW Document]. URL https://minorplanetcenter.net/iau/info/UValue.htm 1 (accessed 3.7.18).

MMA Design LLC, 2014. HaWK ${ }^{\mathrm{TM}}$ Solar Array [WWW Document]. URL http://mmadesignllc.com/products/hawk-solararrays/ (accessed 1.16.18).

Morbidelli, A., Jedicke, R., Bottke, W.F., Michel, P., Tedesco, E.F., 2002. From Magnitudes to Diameters: the Albedo Distribution of Near Earth Objects and the Earth Collision Hazard. Icarus 158 , 329-342. https://doi.org/10.1006/icar.2002.6887

Nanosatellite \& CubeSat Database, 2017. Nanosatellite Database [WWW Document]. URL http://www.nanosats.eu/ (accessed 12.20.17).

NASA, 2017. The Compact Hyperspectral AberrationCorrected Platform (CHAP), an Instrument for Microspacecraft.

NASA, 2015a. Wide-Field InfrarRed Survey TelescopeAstrophysics Focused Telescope Assets WFIRSTAFTA 2015 Report.

NASA, 2015b. Iris V2 CubeSat Deep Space Transponder. Pasadena, CA.

NASA, 2015c. Small Spacecraft Technology State of the Art. Moffett Field, CA, USA. https://doi.org/NASA/TP-2015-216648/REV1

NASA, 2013. JPL HORIZONS [WWW Document]. URL http://ssd.jpl.nasa.gov/?horizons (accessed 1.1.18). 
NASA, 2008. JPL Center for Near Earth Object Studies [WWW Document]. URL https://cneos.jpl.nasa.gov/ (accessed 1.1.18).

NASA, 2005. Low-Cost Innovation in Spaceflight - The Near Earth Asteroid Rendezvous (NEAR) Shoemaker Mission, Monographs in Aerospace History. Washington, D.C., USA.

National Research Council, 2013. Vision and Voyages for Planetary Science in the Decade 2013-2022. The National Academies Press, Washington, D.C., USA.

Navarathinam, N., Lee, R., Chesser, H., 2011. Characterization of Lithium-Polymer Batteries for CubeSat Applications. Acta Astronautica 68, 1752-1760.

https://doi.org/10.1016/j.actaastro.2011.02.004

Nervold, A.K., Berk, J., Straub, J., Whalen, D., 2016. A Pathway to Small Satellite Market Growth. Advances in Aerospace Science and Technology $1,14-20$.

Ocean Optics, 2016. Testing the Orion Spacecraft Heat Shield Design [WWW Document]. URL https://oceanoptics.com/testing-orion-spacecraftheat-shield/ (accessed 6.11.18).

Ocean Optics, 2013a. Ocean Optics STS-UV [WWW Document].

URL https://oceanoptics.com/product/sts-uvmicrospectrometer/ (accessed 1.17.18).

Ocean Optics, 2013b. Ocean Optics STS Series [WWW Document]. URL https://oceanoptics.com/product-category/stsseries/ (accessed 3.20.18).

Palo, S., Stafford, G., Hoskins, A., 2013. An Agile Multi-use Nano Star Camera for Constellation Applications, in: Small Satellite Conference.

Pilbratt, G.L., 2001. The Herschel Mission, Scientific Objectives, and this Meeting, European Space Agency, (Special Publication) ESA SP.

Planetary Resources, 2018. Planetary Resources Launches Latest Spacecraft in Advance of Space Resource Exploration Mission [WWW Document]. URL https://www.planetaryresources.com/2018/01/pla netary-resources-launches-latest-spacecraft-inadvance-of-space-resource-exploration-mission/ (accessed 3.15.18).

Planetary Resources, 2017. Arkyd 6B Spacecraft:
Orbital Debris Assessment Report. Redmond, WA, USA.

Poghosyan, A., Golkar, A., 2017. CubeSat Evolution: Analyzing CubeSat Capabilities for Conducting Science Missions. Progress in Aerospace Sciences 88 , 59-83. https://doi.org/https://doi.org/10.1016/j.paerosci.2 016.11 .002

Poincaré, H., Magini, R., 1899. Les Méthodes Nouvelles de la Mécanique Céleste. Il Nuovo Cimento. https://doi.org/10.1007/BF02742713

Pournelle, R., 2014. Deployment of Cubesats and Small Satellites from the International Space Station, in: Proceedings of the International Astronautical Congress, IAC. pp. 3736-3739.

Praks, J., Kestila, A., Tikka, T., Leppinen, H., Khurshid, O., Hallikainen, M., 2015. AALTO-1 earth observation cubesat mission - Educational outcomes, in: International Geoscience and Remote Sensing Symposium (IGARSS). https://doi.org/10.1109/IGARSS.2015.7326023

Prusti, T., de Bruijne, J.H.J., Brown, A.G.A., Vallenari, A., 2016. The Gaia Mission. Astronomy \& Astrophysics. https://doi.org/10.1051/00046361/201629272

Radius Space, 2016. Radius Space 1U-12U Structures [WWW Document]. URL http://www.radiusspace.com (accessed 1.3.18).

Reddy, V., Dunn, T.L., Thomas, C.A., Moskovitz, N.A., Burbine, T.H., 2015. Mineralogy and Surface Composition of Asteroids. Asteroids IV 43-64. https://doi.org/10.2458/azu_uapress_9780816532 131-ch003

Riedel, J.E., Bhaskaran, S., Desai, S., Hand, D., Kennedy, B., McElrath, T., Ryne, M., 2000. Deep Space 1 Technology Validation Report Autonomous Optical Navigation (AutoNav). Pasadena, CA, USA.

Schoolcraft, J., Klesh, A., Werne, T., 2016. MarCO: Interplanetary Mission Development on a CubeSat Scale, in: AIAA SpaceOps Conference. pp. 1-8. https://doi.org/10.2514/6.2016-2491

SCS Space, 2017. SCS Gecko Imager. Somerset West, South Africa.

Selva, D., Krejci, D., 2013. Preliminary Assessment of Performance and Cost of a Cubesat Component of the Earth Science Decadal Survey. Conference on 
Small Satellites.

Sergeyevsky, A., Snyder, G., Cunniff, R., 1983. Interplanetary Mission Design Handbook, Volume I, Part 2. Earth to Mars Ballistic Mission Opportunities, 1990-2005, NASA. Pasadena, CA, USA.

Simó, C., 1990. On the Analytical and Numerical Approximation of Invariant Manifolds, in: Les Méthodes Modernes de La Mecánique Céleste.

Singer, J., Pelfrey, J., Norris, G., 2017. Secondary Payload Opportunities on NASA's Space Launch System (SLS) Enable Science and Deep Space Exploration, in: Space Operations: Contributions from the Global Community. Springer, pp. 207220. https://doi.org/10.1007/978-3-319-51941-8

Space Telescope Science Institute, 2004. James Webb Space Telescope Project. Greenbelt, MD, USA.

SpaceNews, 2017a. Japanese Startup Raises \$7.3 Million for SmallSat Antenna Sharing Service [WWW Document]. URL http://spacenews.com/japanese-startup-raises-7-3million-for-smallsat-antenna-sharing-service/ (accessed 3.29.18).

SpaceNews, 2017b. British Startup Offers Low-cost CubeSat Services [WWW Document]. URL http://spacenews.com/british-startup-offers-lowcost-cubesat-services/ (accessed 3.29.18).

Springmann, J.C., Kempke, B.P., Cutler, J.W., Bahcivan, H., 2012. Development and Initial Operations of the RAX-2 Cubesat, in: Small Satellites, Systems and Services Symposium. Portoroz, Slovenia, pp. 1-11. https://doi.org/10.1128/MCB.06323-11

Straub, J., 2012. CubeSats: A Low-cost, Very Highreturn Space Technolog, in: AIAA Reinventing Space Conference. Los Angeles, CA, USA.

Swartwout, M., 2013. The First One Hundred CubeSats: A Statistical Look. Journal of Small Satellites 2, 213-233.

Szebehely, V., Jefferys, W.H., 1967. Theory of Orbits: The Restricted Problem of Three Bodies, Academic Press. Elsevier Ltd, New York, NY, USA. https://doi.org/10.1119/1.1974535

Tauber, J.A., 2004. The Planck Mission. Advances in Space Research. https://doi.org/10.1016/j.asr.2003.05.025
Taylor, B., Underwood, C., Dyer, A., Ashton, C., Rason, S., Browning, J., 2011. The Micro Radiation Environment Monitor (MuREM) and SSTL Radiation Monitor (SSTL RM) on TechDemoSat1, in: Radiation Effects on Component and Systems Conference. Institute of Electrical and Electronics Engineers Inc., Sevilla, Spain, pp. 1060-1065.

https://doi.org/10.1109/TNS.2012.2202689

The University of Michigan, 2009. Electrical Power System Overview - RAX.

Thoth Technology, 2013. Thoth Technology Argus IR Spectrometers [WWW Document]. URL http://thothx.com/productcategory/spectrometers/ (accessed 1.17.18).

VACCO Industries, 2015. JPL MarCO Micro CubeSat Propulsion System [WWW Document]. URL http://www.cubesat-propulsion.com/jpl-marcomicro-propulsion-system/ (accessed 12.20.17).

VORAGO Technologies, 2018. Radiation-hardened Solutions for CubeSats, in: 7th Interplanetary CubeSat Workshop. Paris, France.

Wachche, S., Marne, A., Singare, S., Naik, P., Bhide, O., Chaudhari, G., Vartak, P., Pendse, S., Tadwalkar, C., 2014. Thermal Modeling and Simulation of a Pico-Satellite Using Finite Element Method, in: R., P., S., M., R., G., B.L., F. (Eds.), 5th International Conference on Thermal Process Modeling and Computer Simulation, ICTPMCS 2014. ASM International, Pune, India, pp. 65-74.

Walker, R., Koschny, D., Bramanti, C., Cdf, E.S.A., 2017. Miniaturised Asteroid Remote Geophysical Observer (M-ARGO): A Stand-alone Deep Space CubeSat System for Low- cost Science and Exploration Missions, in: 6th Interplanetary CubeSat Workshop. Cambridge, UK.

Wiesel, W.E., 2003. Modern Orbit Determination, 2nd ed. Aphelion Press, Beavercreek, OH, USA.

Xia, X., Sun, G., Zhang, K., Wu, S., Wang, T., Xia, L., Liu, S., 2017. NanoSats/CubeSats ADCS Survey, in: Proceedings of the 29th Chinese Control and Decision Conference. pp. 5151-5158. https://doi.org/10.1109/CCDC.2017.7979410 
2018-11-10

\title{
Asteroid flyby opportunities using semi-autonomous CubeSats: mission design and science opportunities
}

\author{
Machuca, Pablo
}

Elsevier

Machuca P, Sanchez JP, Greenland S. (2019) Asteroid flyby opportunities using semi-autonomous CubeSats: mission design and science opportunities. Planetary and Space

Science, Volume 165, January 2019, pp. 179-193

https://doi.org/10.1016/j.pss.2018.11.002

Downloaded from Cranfield Library Services E-Repository 